\title{
Adaptive Type-2 Fuzzy Neural-Network Control for Teleoperation Systems with Delay and Uncertainties
}

\author{
Parham M. Kebria, Student Member, IEEE, Abbas Khosravi, Member, IEEE, Saeid Nahavandi, Senior \\ Member, IEEE, Dongrui Wu, Senior Member, IEEE, and Fernando Bello
}

\begin{abstract}
Interacting with human operators, remote environment and communication networks, teleoperation systems are considerably suffering from complexities and uncertainties. Managing these is of paramount importance for safe and smooth performance of teleoperation systems. Amongst the countless solutions developed by researchers, type-2 fuzzy algorithms have shown an outstanding performance in modelling complex systems and tackling uncertainties. Moreover, artificial neural networks are well known for their adaptive learning potentials. This paper proposes an adaptive interval type-2 fuzzy neural-network control scheme for teleoperation systems with time-varying delays and uncertainties. The type-2 fuzzy models are developed based on the experimental data collected from a teleoperation setup over a local computer network. However, the resulted controller is evaluated on an intercontinental communication network through the Internet between Australia and Scotland. Moreover, the slave robot and the remote workspace are completely different and unforeseen. Stability and performance of the proposed control is analysed by Lyapunov-Krasovskii method. Comprehensive comparative studies demonstrate that the proposed controller outperforms traditional techniques in experimental evaluations.
\end{abstract}

Index Terms-Teleoperation systems, type-2 fuzzy models, neural-network, adaptive control, uncertainties, time-delay.

\section{INTRODUCTION}

$\mathbf{T}$ ELEOPERATION systems have provided the humankind with a variety of great applications, especially in space explorations and medicine [1]-[5]. Involving various complex technologies, teleoperation systems severely suffer from uncertainties and nonlinearities [6]. Particularly, uncertainties in communication networks, human operators' and robots' dynamics, and remote environment are the major concerns in teleoperation applications. Therefore, controller design for such systems has become a problematic task that a large amount of research has been committed to address these issues [7]. Amongst the proposed solutions for control of teleoperation systems, control methods based on computational intelligence have outperformed the traditional methodologies, in terms of dealing with complexities and uncertainties [8].

Introduced by Lotfi Zadeh [9]-[12], fuzzy computation approaches have undoubtedly shown a strong capability in the

P. M. Kebria, A. Khosravi, and S. Nahavandi are with the Institute for Intelligent Systems Research and Innovations (IISRI), Deakin University, Waurn Ponds, VIC 3216, Australia (e-mail: kebria@deakin.edu.au).

D. Wu is with the Key Laboratory of the Ministry of Education for Image Processing and Intelligent Control, School of Automation, Huazhong University of Science and Technology, Wuhan, China (e-mail: drwu@hust.edu.cn).

Fernando Bello is with the Centre for Engagement and Simulation Science, Imperial College London, Chelsea and Westminster Hospital (Academic Surgery), London, UK (e-mail: F.Bello@imperial.ac.uk). modelling of complex systems. Based on their universal approximations, fuzzy models are extremely useful in controller design for complex and nonlinear systems [13]-[22], decisionmaking and forecasting [23]-[26], and disturbance rejection [27]-[30]. Basically, fuzzy models describe a considered system's dynamical behaviour as a composition of several IFTHEN rules. Among the fuzzy modelling approaches, TakagiSugeno (T-S) [27] and Mamdani [31] are the most popular methods. Traditional fuzzy models, also known as type-1, assign crisp fuzzy membership functions on their variables [32]. Consequently, the system's model and performance of the controller designed based on this model will be vulnerable and sensitive to uncertainties [33]. In contrast, type-2 fuzzy (T2F) methodologies [34]-[36] have demonstrated a promising effectiveness in dealing with uncertainties [37]-[41]. Type-2 fuzzy models may have type-2 membership functions for either antecedents or consequents [38], [39], [42].

In the field of teleoperation systems, researchers have proposed a two-layer fuzzy strategy on a remote mobile robot to tackle the communications failures [43]. In this approach, outputs of each fuzzy rule are input of the immediately next rule and are called the intermediate fuzzy variables. Hence, the resulted fuzzy controller has a network structure with a robust performance [44]. Reference [45] has considered a multi robot teleoperation system and proposed an adaptive fuzzy control strategy to compensate for the communication delay modelled by Markov process. They have considered the master and slave robots dynamically decoupled, which reduces the applicability of the proposed control algorithm. Combining with traditional sliding-mode control approaches, [46] has proposed a finitetime fuzzy controller for a teleoperation system, however, under constant time-delay. T-S fuzzy controller proposed in [47] is based on the systems' parameters, and therefore, does not cope with model uncertainties. Researchers in [48] have developed an adaptive fuzzy solution, in which a switching algorithm is proposed for delay considerations. However, no uncertainty is taken into account and the resulted control torque signals are chattering, which degrades the practicality. The state convergent fuzzy controller in [49] is designed based on the linear model of the teleoperation system and constant time-delay. Although the fuzzy controller proposed in [50], [51] have reduced the chattering in torque signals, it is applicable only for constant delays and no uncertainty is considered. [52] has recently proposed a fuzzy observer-based control for teleoperation systems with time-delay.

Meanwhile, control solutions developed based on type-2 fuzzy models are more reliable and effective. For example, 
[53] has proposed a type-2 fuzzy controller combined with a wavelet neural-network (NN). However, they have considered type-2 fuzzy membership functions only for the antecedents, but crisp functions for the consequents, which decreases the robustness of the controller when facing uncertainties and time-delays. Researchers in [54] proposed a controller based on a data-driven type- 2 fuzzy model of a teleoperation system to deal with time-delay and uncertainties. They have also applied Markov technique to model the network delay. Although closed-loop stability of the proposed control is proved by Lyapunov-Krasovskii and Linear Matrix Inequality (LMI), its performance and error convergence depends on the systems' parameters and time-derivatives of the delay functions, which are not necessarily available. A similar methodology has also been applied in [55]. Moreover, the experimental evaluation carried out in [53] and [54] was on two simple and similar devices. However, in real-world applications, the master and slave robots are generally dissimilar with completely different workspace and dynamics. This dissimilarity introduces additional uncertainties to teleoperation systems, and complicates the control design for each robot. Additionally, they have not considered human operator's and environment's dynamics that involve much uncertainties in the teleoperation dynamic analyses and performance [56]-[58].

On the other side, learning capabilities of neural networks further empowers type-2 fuzzy NN-based controllers to adaptively deal with external disturbances and internal uncertainties [59]-[67]. Many research studies have validated the functionality of adaptive NN-based control algorithms in complex real-world applications, including teleoperation systems [68]-[71]. Unknown remote workspace and communication characteristics are treated as the main sources of uncertainties in teleoperation systems. Hence, an adaptive control strategy with the ability of learning unknown dynamics, and also, dealing with uncertainties is required to protect the stability and performance of a teleoperation system. Therefore, investigation for an effective controller ensuring a teleoperation performance while confronting uncertainties and latencies is the main motivation for the current article. Considering difficulties encountered in teleoperation systems, this paper aims to develop an efficient control solution applicable to real-world applications.

This study proposes a control methodology for teleoperation systems that not only accomplishes the desired task in the remote workspace, it also prevents instability and failures. The proposed controller takes the advantages of T2F techniques to deal with time-varying delays and uncertainties. Moreover, an NN-based online adaptation algorithm is developed to simultaneously tune the T2F models. Chiefly, main contributions of this study are:

- Interval type-2 fuzzy (IT2F) modelling for Internet-based teleoperation systems. A real-world experimental teleoperation system has been considered for data collection. The collected data is then used for derivation of $\mathrm{T} 2 \mathrm{~F}$ models for increasing the fuzziness in antecedents and consequents. This way enables the controller to handle further uncertainties.

- It is worth to mention that this study considers two different teleoperation configurations for each data collection and validation purposes. The main reason for this consideration is two folded: first, for an efficient way for collecting experimental data; and second, validation of the proposed controller in a more realistic application that involves an intercontinental Internet communication and unforeseen workspace. This incurs uncertainties and time-delays that a real-world teleoperation experiences. Furthermore, the results demonstrate the effectiveness and practicality of the proposed control in real-world applications.

- The considered teleoperation system for experimental data collection consists of two Phantom Omni Haptic devices on two different desktop computers connected through a Local Area Network (LAN), Figure 1. Notably, this teleoperation setup is only used in the data collection phase for $\mathrm{T} 2 \mathrm{~F}$ modelling purposes. But the proposed control algorithm is then evaluated on a different teleoperation configuration. Slave robot of the target teleoperation system is a virtual dynamic model of the UR5 manipulator. The slave system is located in Scotland receiving commands from the master system in Australia (Figure 7). The master system communicates over a $4 \mathrm{G}$ mobile network, while the slave system is connected to the cable Internet.

- The proposed controller is empowered by an adaptive $\mathrm{NN}$ that concurrently updates the parameters of the T2F model. To not getting stuck in local optima, the online adaptation laws are derived by Lyapunov-Krasovskii approach. This also justifies the performance of the proposed Type-2 fuzzy neural network (T2FNN) controller under time-varying delays and uncertainties.

Furthermore, to illustrate the superiority of the proposed control solution over the previous similar studies, experimental evaluations are also executed and compared with the control algorithms developed in [53] and [54].

This paper is organised as follows: Section II describes the T2F modelling process of the Internet-based teleoperation system. Section III explains the derivation of the proposed adaptive T2FNN control algorithm for the teleoperation system. Performance of the proposed control methodology is experimentally evaluated in Section IV, followed by conclusion remarks and discussions in Section V.

\section{TYPe-2 FuZZy MODELLING OF INTERNET-BASED TELEOPERATION SYSTEMS}

Dynamics of a master/slave robotic teleoperation system has the following general form (Figure 2):

$$
\begin{array}{r}
M_{i}\left(q_{i}\right) \ddot{q}_{i}+C_{i}\left(q_{i}, \dot{q}_{i}\right)+g_{i}\left(q_{i}\right)+\mu_{i}\left(\dot{q}_{i}, q_{i}\right) \\
=\tau_{i}+J_{i}^{T}\left(q_{i}\right)\left(f_{j}\right)+\sigma_{j}(t)
\end{array}
$$

where, $i \in\{m, s\}$ indicates the master and slave systems, respectively. $j \in\{h, e\}$ refers to the human operator and remote environment. $q_{i} \in \mathbb{R}^{n}, \dot{q}_{i}$ and $\ddot{q}_{i}$ denote the joints' angular position, velocity and acceleration of the corresponding robot, respectively. $M_{i} \in \mathbb{R}^{n \times n}$ is the inertia matrix, $C_{i} \in \mathbb{R}^{n \times n}$ the Coriolis and centripetal terms, and $g_{i} \in \mathbb{R}^{n}$ the 


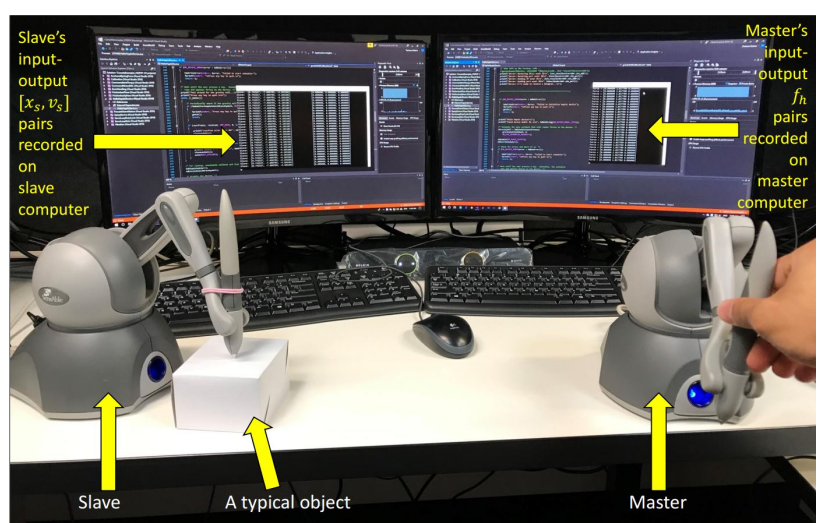

Fig. 1. The teleoperation setup with two Phantom Omni Haptic devices used for data collection. It should be mentioned that the sampling time of the whole experiment, including data collection and control implementation is 0.001 second $(1 \mathrm{kHz})$. A very similar setup has been used in [54] for both modelling and evaluations. In this study, experimental evaluations are implemented and executed on a different and more complicated setup with a real intercontinental communication (Figure 7).

gravitational vector of the corresponding robot, respectively. $\mu_{i} \in \mathbb{R}^{n}$ stands for the unknown viscous and Coulomb friction vector. $\tau_{i} \in \mathbb{R}^{n}$ is the input torques of the robots. $f_{j} \in \mathbb{R}^{6}$ is the force imposed by either the human operator or remote environment. $\sigma_{j} \in \mathbb{R}^{n}$ is the external disturbances. Noticeably, $n \in \mathbb{N}$ indicates the corresponding robot's degree of freedom (DoF). $J_{i}$ is the Jacobian matrix of the robots that relates the Cartesian and joint space variables of each robot [72], [73]. Jacobian calculations is one of the major uncertain and challenging concerns in robots' dynamic modelling. Moreover, dissimilarity between the master and slave robots introduces further difficulties [74]. However, in [54] the master and slave robots are identical. In this study, we considered the similar teleoperation setup as [54] for data collection (Figure 1). But the outcome will be evaluated on a more realistic teleoperation configuration (Figure 7). Moreover, the human operator and remote workspace are generally modelled as nonhomogeneous mass-spring-damper forces [56]:

$$
f_{j}=f_{j 0}+M_{j} \ddot{\mathbf{x}}_{i}+B_{j} \dot{\mathbf{x}}_{i}+K_{j} \mathbf{x}_{i}
$$

in which, $j=h$ when $i=m$, and $j=e$ when $i=s$. $f_{j 0} \in \mathbb{R}^{6}$ is the non-homogeneous term, and $M_{j}, B_{j}$ and $K_{j}$ are inertia, damping and stiffness coefficient matrices, respectively. It should be mentioned that all the matrices, vectors and coefficients in equations (1) and (2) are unknown and uncertain. Moreover, $\mathbf{x}_{i}=\mathbf{p}_{i}\left(q_{i}\right), \mathbf{p}_{i}():. \mathbb{R}^{n} \rightarrow \mathbb{R}^{6}$, $\dot{\mathbf{x}}=J_{i}\left(q_{i}\right) \dot{q}_{i}$ and $\ddot{\mathbf{x}}=J_{i}\left(q_{i}\right) \ddot{q}_{i}+\dot{J}_{i}\left(q_{i}\right) \dot{q}_{i}$ are the Cartesian position $\left(\mathbf{p}_{i}\left(q_{i}\right)\right.$ is forward kinematics), velocity and acceleration of each robot in its workspace, respectively. More details of the totally uncertain dynamics of the teleoperation system (1) coupled with (2) is found in [56]. Here, the coupled dynamics of (1) and (2) for each sides of the teleoperation system is being expressed as:

$$
\mathcal{M}_{i} \ddot{\mathbf{x}}_{i}+\mathcal{C}_{i}\left(\mathbf{x}_{i}, \dot{\mathbf{x}}_{i}\right)+\mathcal{G}_{i}\left(\mathbf{x}_{i}\right)=\tau_{i}+\mathbf{f}_{j}+\sigma_{j}(t)
$$

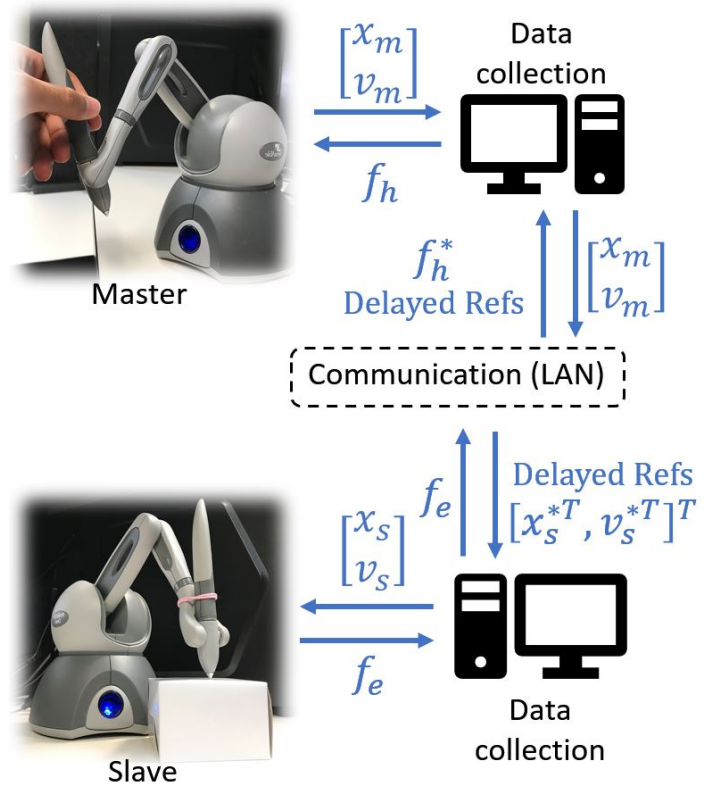

Fig. 2. The considered teleoperation configuration for data collection.

in which

$$
\begin{aligned}
\mathcal{M}_{i} & =M_{i} J_{i}^{\dagger}-J_{i}^{T} M_{j} \\
\mathcal{C}_{i}\left(\mathbf{x}_{i}, \dot{\mathbf{x}}_{i}\right) & =C_{i}+\mu_{i}-\left(M_{i} J_{i}^{\dagger} \dot{J}_{i} J_{i}^{\dagger}+J_{i}^{T} B_{j}\right) \dot{\mathbf{x}}_{i} \\
\mathcal{G}_{i}\left(\mathbf{x}_{i}\right) & =g_{i}-J_{i}^{T} K_{j} \mathbf{x}_{i} \\
\mathbf{f}_{j} & =J_{i}^{T} f_{j} \\
J_{i}^{\dagger} & =\left(J_{i}^{T} J_{i}\right)^{-1} J_{i}^{T}
\end{aligned}
$$

It should be noted that all the matrices and coefficients above are uncertain and partially unknown. However, due to physical limitations and structures, there are upper and lower bounds for every unknown parameter considered as uncertainty intervals, including $\left[\underline{\mathcal{M}}_{i}, \overline{\mathcal{M}}_{i}\right],\left[\underline{\mathcal{C}}_{i}, \overline{\mathcal{C}}_{i}\right],\left[\underline{\mathcal{G}}_{i}, \overline{\mathcal{G}}_{i}\right]$, and $\left[\underline{\sigma}_{j}, \bar{\sigma}_{j}\right]$.

In most of the real-world teleoperation applications, the slave robot is desired to track the master's position $\left(\mathbf{x}_{m}\right)$ and velocity $\left(\mathbf{v}_{m}\right)$, and the master device should reflect the force imposed on the slave robot while interacting with the remote environment $\left(\mathbf{f}_{e}\right)$. To this end, the required Cartesian and haptic information are being transmitted through the communication channel during the teleoperation process. Consequently, latencies and uncertainties induced by the communication network will delay the transmission procedure. In other words, desired signals $\mathbf{x}_{s}^{*}, \mathbf{v}_{s}^{*}$ and $\mathbf{f}_{h}^{*}$ are considered as:

$$
\begin{aligned}
& \mathbf{x}_{s}^{*}(t)=\mathbf{x}_{m}\left(t-d_{f}(t)\right) \\
& \mathbf{v}_{s}^{*}(t)=\mathbf{v}_{m}\left(t-d_{f}(t)\right) \\
& \mathbf{f}_{h}^{*}(t)=\mathbf{f}_{e}\left(t-d_{b}(t)\right)
\end{aligned}
$$

where, $d_{f}(t)$ and $d_{b}(t)$ are time-varying delays through the forward and backward communication channels, respectively.

To tackle these uncertainties, we utilise Interval Type-2 Fuzzy (IT2F) modelling approach. A sufficiently large number of input-output pairs are collected from the teleoperation setup in Figure 1. At the master side, $\mathbf{f}_{h}^{*}(k T)$ is considered as the input and $\mathbf{f}_{h}(k T)$ as the output. Whereas, $\mathbf{x}_{s}^{*}(k T)$ and 


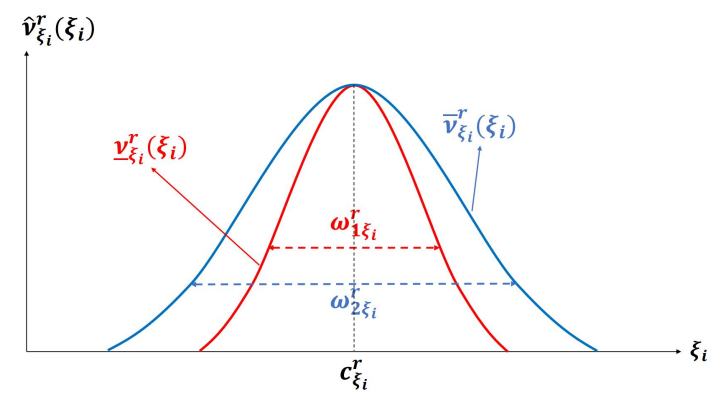

Fig. 3. The uncertain Gaussian function considered for every T2F membership function.

$\mathbf{v}_{s}^{*}(k T)=\dot{\mathbf{x}}_{s}^{*}(k T)$ are treated as the inputs, and $\mathbf{x}_{s}(k T)$ and $\mathbf{v}_{s}(k T)=\dot{\mathbf{x}}_{s}(k T)$ as the outputs of the slave system, with the sampling period $T=1 \mathrm{~ms}$ and $k \in \mathbb{N}$. Hereafter, we drop the sampling argument $(k T)$ and denote the input-output pairs by $\chi_{s}=\left[\mathbf{x}_{s}^{T}, \mathbf{v}_{s}^{T}\right]^{T}$ and $\chi_{s}^{*}=\left[\mathbf{x}_{s}^{* T}, \mathbf{v}_{s}^{* T}\right]^{T}$, for simplicity and saving space. IT2F approach is applied on every inputoutput pair to derive the mapping model between the positionvelocity/force reference and output signals.

Considering the delayed reference signal $\mathbf{f}_{h}^{*}$ and the haptic output $\mathbf{f}_{h}$ for the master system, the desired and actual position-velocity $\chi_{s}^{*}$ and $\chi_{s}$ for the slave system, IT2F model describes the teleoperation system in the form of several IFTHEN rules like:

$$
\begin{aligned}
& \text { Rule } r_{s}: \mathrm{IF} \mathbf{x}_{s}^{*} \in S_{x}^{r} \text {, and } \mathbf{v}_{s}^{*} \in S_{v}^{r} \\
& \text { THEN }: \hat{\chi}_{s}^{r}=\hat{a}_{s 0}^{r}+\hat{A}_{x}^{r} \mathbf{x}_{s}^{*}+\hat{A}_{v}^{r} \mathbf{v}_{s}^{*}
\end{aligned}
$$

for the slave system, and

$$
\begin{aligned}
& \text { Rule } r_{m}: \mathrm{IF} \mathbf{f}_{h}^{*} \in S_{f}^{r} \\
& \text { THEN }: \hat{\mathbf{f}}_{h}^{r}=\hat{a}_{m 0}^{r}+\hat{A}_{f}^{r} \mathbf{f}_{h}^{*}
\end{aligned}
$$

for the master system, where $r_{i}=1, \ldots, R_{i}, R_{i}$ is the total number of rules for each system. $\hat{A}_{\xi}^{r}, \xi \in\{x, v, f\}$ are diagonal matrices of $\left[\hat{a}_{\xi 1}^{r}, \ldots, \hat{a}_{\xi 6}^{r}\right]^{T}$ and $\hat{a}_{\xi n}^{r} \subseteq\left[\underline{a}_{\xi n}^{r}, \bar{a}_{\xi n}^{r}\right], n=1, \ldots, 6$ and $\hat{a}_{i 0}^{r} \subseteq\left[\underline{a}_{i 0}^{r}, \bar{a}_{i 0}^{r}\right]$ are uncertain interval coefficients. These parameters are basically related to the dynamics characteristics of the robots. The upper and lower bounds of these parameters indicates the range of the uncertainties in the dynamics of the systems. In this study, we applied the mathematical modelling tools and knowledges developed in [72], [73] to derive numerical estimations of those parameters. Accordingly, $\hat{\chi}_{s}^{r} \subseteq\left[\underline{\chi}_{s}^{r}, \bar{\chi}_{s}^{r}\right]$ and $\hat{\mathbf{f}}_{h}^{r} \subseteq\left[\underline{\mathbf{f}}_{h}^{r}, \overline{\mathbf{f}}_{h}^{r}\right]$ in which:

$$
\begin{array}{lll}
\underline{\chi}_{s}^{r}=\underline{a}_{s 0}^{r}+\underline{A}_{x}^{r} \mathbf{x}_{s}^{*} & , \quad \bar{\chi}_{s}^{r}=\bar{a}_{s 0}^{r}+\bar{A}_{x}^{r} \mathbf{x}_{s}^{*} \\
\underline{\mathbf{f}}_{h}^{r}=\underline{a}_{m 0}^{r}+\underline{A}_{f}^{r} \mathbf{f}_{h}^{*} & , \quad \overline{\mathbf{f}}_{h}^{r}=\bar{a}_{m 0}^{r}+\bar{A}_{f}^{r} \mathbf{f}_{h}^{*}
\end{array}
$$

$S_{x}^{r}, \quad S_{v}^{r}$ and $S_{f}^{r}$ are the IT2F sets with IT2F membership functions respectively denoted by $\hat{\nu}_{x}^{r}\left(\mathbf{x}_{s}\right)=$ $\left[\underline{\nu}_{x}^{r}\left(\mathbf{x}_{s}\right), \bar{\nu}_{x}^{r}\left(\mathbf{x}_{s}\right)\right], \hat{\nu}_{v}^{r}\left(\mathbf{v}_{s}\right)=\left[\underline{\nu}_{v}^{r}\left(\mathbf{v}_{s}\right), \bar{\nu}_{v}^{r}\left(\mathbf{v}_{s}\right)\right]$ and $\hat{\nu}_{f}^{r}\left(\mathbf{f}_{h}\right)=$ $\left[\underline{\nu}_{f}^{r}\left(\mathbf{f}_{h}\right), \bar{\nu}_{f}^{r}\left(\mathbf{f}_{h}\right)\right]$. Illustrated in Figure 3 , Gaussian functions with uncertain standard deviation and mean are considered

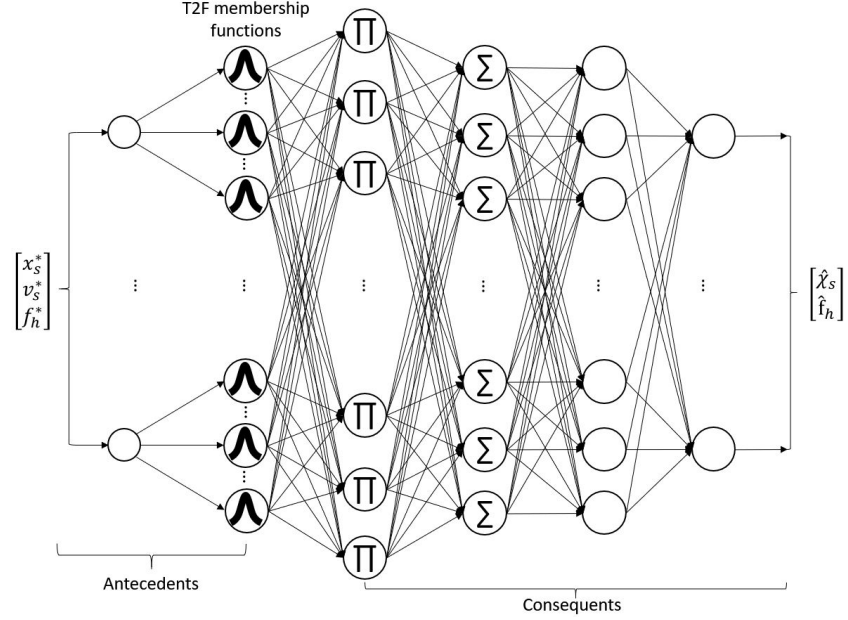

Fig. 4. Structure of the IT2FNN models.

for every IT2F membership function $0 \leq \underline{\nu}_{\xi_{i}}^{r}\left(\xi_{i}\right) \leq \bar{\nu}_{\xi_{i}}^{r}\left(\xi_{i}\right) \leq$ $1, \xi_{i} \in\left\{\mathbf{x}_{s}, \mathbf{v}_{s}, \mathbf{f}_{h}\right\}$ :

$$
\begin{aligned}
& \underline{\nu}_{\xi_{i}}^{r}\left(\xi_{i}\right)=\exp \left(-\frac{1}{2}\left(\frac{\xi_{i}-c_{\xi_{i}}^{r}}{\omega_{1 \xi_{i}}^{r}}\right)^{2}\right) \\
& \bar{\nu}_{\xi_{i}}^{r}\left(\xi_{i}\right)=\exp \left(-\frac{1}{2}\left(\frac{\xi_{i}-c_{\xi_{i}}^{r}}{\omega_{2 \xi_{i}}^{r}}\right)^{2}\right)
\end{aligned}
$$

As depicted in Figure 3, $\omega_{1 \xi_{i}}^{r}$ and $\omega_{2 \xi_{i}}^{r}$ are the uncertain standard deviations of the lower and upper Gaussian membership functions $\underline{\nu}_{\xi_{i}}^{r}\left(\xi_{i}\right)$ and $\bar{\nu}_{\xi_{i}}^{r}\left(\xi_{i}\right)$, respectively, with the uncertain means $c_{\xi_{i}}^{r}$. Noticeably, these parameters are being updated by the adaptive NN-based algorithm that will be developed in the next section.

Applying the product operator, the lower and upper functions of each rule in (5) are derived:

$$
\begin{aligned}
& \underline{\eta}_{s}^{r}\left(\chi_{s}\right)=\underline{\nu}_{x}^{r}\left(\mathbf{x}_{s}\right) \times \underline{\nu}_{v}^{r}\left(\mathbf{v}_{s}\right) \\
& \bar{\eta}_{s}^{r}\left(\chi_{s}\right)=\bar{\nu}_{x}^{r}\left(\mathbf{x}_{s}\right) \times \bar{\nu}_{v}^{r}\left(\mathbf{v}_{s}\right)
\end{aligned}
$$

Combining the $R_{i}$ IT2F rules and applying the type reduction method proposed in [75], outputs of the rules are derived as:

$$
\begin{gathered}
\hat{\chi}_{s}=\frac{1}{2}\left(\frac{\sum_{r=1}^{R_{s}} \underline{\eta}_{s}^{r}\left(\chi_{s}\right) \underline{\chi}_{s}^{r}}{\sum_{r=1}^{R_{s}} \underline{\eta}_{s}^{r}\left(\chi_{s}\right)}+\frac{\sum_{r=1}^{R_{s}} \bar{\eta}_{s}^{r}\left(\chi_{s}\right) \bar{\chi}_{s}^{r}}{\sum_{r=1}^{R_{s}} \bar{\eta}_{s}^{r}\left(\chi_{s}\right)}\right) \\
\hat{\mathbf{f}}_{h}=\frac{1}{2}\left(\frac{\sum_{r=1}^{R_{m}} \underline{\nu}_{f}^{r}\left(\mathbf{f}_{h}\right) \underline{\mathbf{f}}_{h}^{r}}{\sum_{r=1}^{R_{m}} \underline{\nu}_{f}^{r}\left(\mathbf{f}_{h}\right)}+\frac{\sum_{r=1}^{R_{m}} \bar{\nu}_{f}^{r}\left(\mathbf{f}_{h}\right) \overline{\mathbf{f}}_{h}^{r}}{\sum_{r=1}^{R_{m}} \bar{\nu}_{f}^{r}\left(\mathbf{f}_{h}\right)}\right)
\end{gathered}
$$

Structure of the resulted IT2FNN is shown in Figure 4. The initial values for the parameters and above, especially the mean of the Gaussian membership function, have been set by the previous calculations and tools developed in [72]. Moreover, in the next section, an adaptive algorithm is proposed for updating the network parameters of the IT2FNN model, including uncertain standard deviations and means 


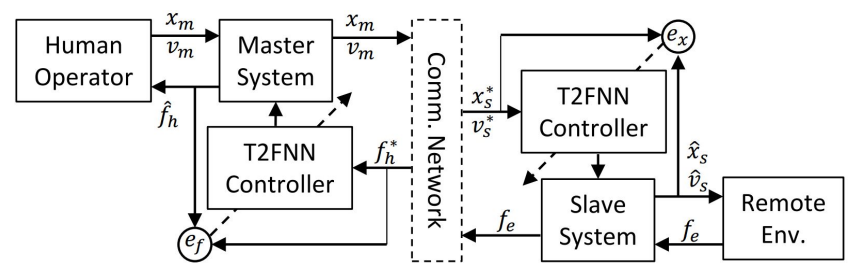

Fig. 5. The considered teleoperation configuration for development and evaluation of the adaptive T2FNN controller.

of the IT2F membership functions. The adaptation laws are based on gradient descent method, however, to prevent from getting stuck in local optima, the updating rules are derived by Lyapunov-Krasovskii functional. In addition, this derivation also guarantees the stability and convergent performance of the proposed controller in the presence of time-delays.

\section{ADAPTIVE T2FNN CONTROLLER}

The teleoperation configuration for development and evaluation of the adaptive T2FNN controller is shown in Figure 5. Illustrating the overall design process, Figure 6 also depicts the flowchart of the proposed adaptive T2FNN control algorithm.

The main goal of this study is to develop a control strategy that robustly minimises the teleoperation errors:

$$
e_{\mathbf{x}}=\mathbf{x}_{s}^{*}-\hat{\mathbf{x}}_{s} \quad, \quad e_{\mathbf{f}}=\mathbf{f}_{h}^{*}-\hat{\mathbf{f}}_{h}
$$

To achieve this goal, a cost function is considered for each the master and slave subsystems:

$$
E_{m}=\frac{1}{2} e_{\mathbf{f}}^{T} e_{\mathbf{f}}=\frac{1}{2}\left\|e_{\mathbf{f}}\right\|^{2} \quad, \quad E_{s}=\frac{1}{2} e_{\mathbf{x}}^{T} e_{\mathbf{x}}=\frac{1}{2}\left\|e_{\mathbf{x}}\right\|^{2}
$$

Adaptation laws for adjustable parameters of the T2FNN controllers should be derived in order to minimise these objective function. This section explains online learning algorithms for the T2FNN controllers for the master and slave systems. We use the subscription $i \in\{m, s\}$ and the notations $\xi \in\left\{\mathbf{x}_{s}, \mathbf{f}_{h}\right\}$ for simplification. Update laws are proposed as follows:

$$
\begin{gathered}
c_{\xi}^{r}(k+1)=\alpha_{i} c_{\xi}^{r}(k)+\gamma_{i}(k) \frac{\partial E_{i}(k)}{\partial c_{\xi}^{r}(k)} \\
\omega_{1 \xi}^{r}(k+1)=\alpha_{i} \omega_{1 \xi}^{r}(k)+\gamma_{i}(k) \frac{\partial E_{i}(k)}{\partial \omega_{1 \xi}^{r}(k)} \\
\omega_{2 \xi}^{r}(k+1)=\alpha_{i} \omega_{2 \xi}^{r}(k)+\gamma_{i}(k) \frac{\partial E_{i}(k)}{\partial \omega_{2 \xi}^{r}(k)}
\end{gathered}
$$

$\alpha_{i}$ and $\gamma_{i}$ are learning rates. To avoid getting stuck in local minima and to increase the efficiency in adaptation [76], the learning rate $\gamma_{i}$ is also considered adaptive:

$$
\dot{\gamma}_{i}=\left(1+\left\|e_{\xi}\right\|\right)\left\|e_{\xi}\right\|
$$

Considering $\Delta k T \rightarrow T$ as the one step ahead, time-derivative

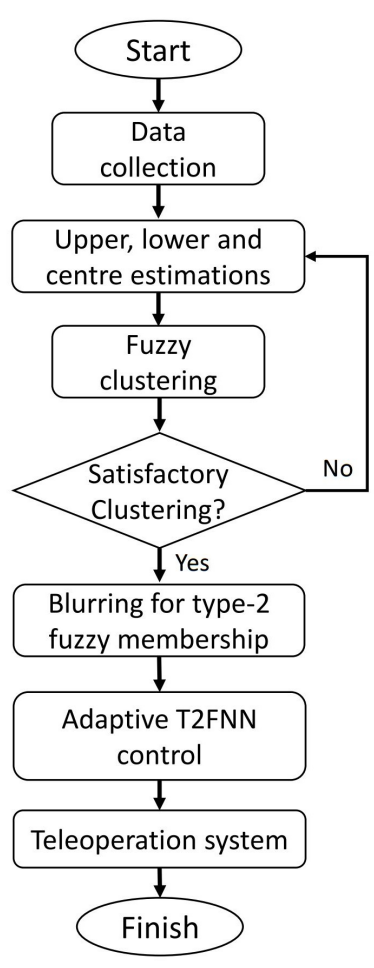

Fig. 6. Flowchart of the proposed adaptive T2FNN controller.

of the adaptive parameters are derived as

$$
\begin{gathered}
\dot{c}_{\xi}^{r}=\left(\alpha_{i}-1\right) c_{\xi}^{r}+\gamma_{i} \frac{\partial E_{i}}{\partial c_{\xi}^{r}} \\
\dot{\omega}_{1 \xi}^{r}=\left(\alpha_{i}-1\right) \omega_{1 \xi}^{r}+\gamma_{i} \frac{\partial E_{i}}{\partial \omega_{1 \xi}^{r}} \\
\dot{\omega}_{2 \xi}^{r}=\left(\alpha_{i}-1\right) \omega_{2 \xi}^{r}+\gamma_{i} \frac{\partial E_{i}}{\partial \omega_{2 \xi}^{r}}
\end{gathered}
$$

Moreover, applying the chain rules:

$$
\begin{aligned}
\frac{\partial E_{i}}{\partial c_{\xi}^{r}} & =\frac{\partial E_{i}}{\partial e_{\xi}} \cdot \frac{\partial e_{\xi}}{\partial \hat{\xi}} \cdot \frac{\partial \hat{\xi}}{\partial c_{\xi}^{r}}=\left(e_{\xi}^{T}\right)(-1) \frac{\partial \hat{\xi}}{\partial c_{\xi}^{r}}=-e_{\xi}^{T} \frac{\partial \hat{\xi}}{\partial c_{\xi}^{r}} \\
\frac{\partial E_{i}}{\partial \omega_{1 \xi}^{r}} & =\frac{\partial E_{i}}{\partial e_{\xi}} \cdot \frac{\partial e_{\xi}}{\partial \hat{\xi}} \cdot \frac{\partial \hat{\xi}}{\partial \omega_{1 \xi}^{r}}=-e_{\xi}^{T} \frac{\partial \hat{\xi}}{\partial \omega_{1 \xi}^{r}} \\
\frac{\partial E_{i}}{\partial \omega_{2 \xi}^{r}} & =\frac{\partial E_{i}}{\partial e_{\xi}} \cdot \frac{\partial e_{\xi}}{\partial \hat{\xi}} \cdot \frac{\partial \hat{\xi}}{\partial \omega_{2 \xi}^{r}}=-e_{\xi}^{T} \frac{\partial \hat{\xi}}{\partial \omega_{2 \xi}^{r}}
\end{aligned}
$$

and

$$
\dot{\hat{\xi}}=\frac{\partial \hat{\xi}}{\partial c_{\xi}^{r}} \dot{c}_{\xi}^{r}+\frac{\partial \hat{\xi}}{\partial \omega_{1 \xi}^{r}} \dot{\omega}_{1 \xi}^{r}+\frac{\partial \hat{\xi}}{\partial \omega_{2 \xi}^{r}} \dot{\omega}_{2 \xi}^{r}+\frac{\partial \hat{\xi}}{\partial \xi^{*}} \dot{\xi}^{*}
$$

It should be noted that although the derivative term $\dot{\xi}^{*}=\dot{\mathbf{f}}_{h}^{*}$ in (17) is not practically available for the master system, it could be calculated by $\dot{\xi}^{*}=\left(\xi^{*}(k T)-\xi^{*}((k-1) T)\right) / T$. It is also known that every force profile should be physically limited and cannot vary much fast due to safety considerations and mechanical limitations [6]-[8]. Hence,

$$
\left\|\dot{\xi}^{\star}\right\| \leq \bar{\Xi}, \quad \bar{\Xi}: \text { a positive constant. }
$$


However, $\dot{\xi}^{*}=\dot{\mathbf{x}}_{s}^{*}=\mathbf{v}_{s}^{*}$ is feasible. Additionally, these timederivative terms are not used in the design procedure, and appear only in theoretical analyses.

Theorem: The T2FNN controller (10) with the adaptation laws (14) and (15), where

$$
\begin{gathered}
\frac{\partial E_{i}}{\partial c_{\xi}^{r}}=\max \left(P_{i 1}, P_{i 2}\right) \\
\frac{\partial E_{i}}{\partial \omega_{1 \xi}^{r}}=\max \left(N_{i 1}, N_{i 2}\right) \\
\frac{\partial E_{i}}{\partial \omega_{2 \xi}^{r}}=\max \left(Q_{i 1}, Q_{i 2}\right)
\end{gathered}
$$

in which $P_{i 1,2}, N_{i 1,2}$ and $Q_{i 1,2}$ are derived in the Proof below, minimises the cost functions (12).

Proof: Considering the Lyapunov-Krasovskii functional:

$$
\begin{aligned}
V_{i}(t) & =\frac{1}{2} e_{\xi}^{T} e_{\xi}+\int_{t-d_{\delta}(t)}^{t} e_{\xi}^{T} e_{\xi} d \theta+\int_{t-d_{\delta}(t)}^{t} \dot{e}_{\xi}^{T} \dot{e}_{\xi} d \theta \\
& +\int_{-D_{\delta}}^{0} \int_{t+\theta}^{t} e_{\xi}^{T} e_{\xi} d \zeta d \theta+\frac{1}{2}\left(\gamma_{i}-\gamma_{i}^{*}\right)^{2}
\end{aligned}
$$

where $\left|d_{\delta}(t)\right|$ is the corresponding communication delay for $\delta \in\{f, b\} . \gamma_{i}^{*}$ is an arbitrary design parameter. We show that a proper solution of (19) makes $\dot{V}_{i}$ negative-definite, and therefore, the online learning algorithm (15) guarantees the asymptotic performance of the T2FNN controllers (10a) and (10b). Taking time-derivatives of $V_{i}$

$$
\begin{aligned}
\dot{V}_{i} & =e_{\xi}^{T} \dot{e}_{\xi}^{T}+\left\|e_{\xi}\right\|^{2}-\left(1-\dot{d}_{\delta}(t)\right)\left\|e_{\xi}\left(t-d_{\delta}(t)\right)\right\|^{2} \\
& +\left\|\dot{e}_{\xi}\right\|^{2}-\left(1-\dot{d}_{\delta}(t)\right)\left\|\dot{e}_{\xi}\left(t-d_{\delta}(t)\right)\right\|^{2}+D_{\delta}\left\|e_{\xi}\right\|^{2} \\
& -\int_{t+\theta}^{t} e_{\xi}^{T}(\xi) e_{\xi}(\xi) d \xi+\dot{\gamma}_{i}\left(\gamma_{i}-\gamma_{i}^{*}\right)
\end{aligned}
$$

where $\left|d_{\delta}(t)\right| \leq D_{\delta}$ and $\dot{d}_{\delta}(t)<1$ are practical assumptions that are experimentally verified (Figure 8 ). Therefore,

$$
\dot{V}_{i}<e_{\xi}^{T} \dot{e}_{\xi}+\left\|e_{\xi}\right\|^{2}\left(1+D_{\delta}\right)+\left\|\dot{e}_{\xi}\right\|^{2}+\dot{\gamma}_{i}\left(\gamma_{i}-\gamma_{i}^{*}\right)
$$

from (18) and knowing that $\dot{e}_{\xi}=\dot{\xi}^{*}-\dot{\hat{\xi}} \leq \bar{\Xi}-\dot{\hat{\xi}}$, then

$$
\dot{V}_{i}<\bar{\Xi}\left\|e_{\xi}\right\|-e_{\xi}^{T} \dot{\hat{\xi}}+\left\|e_{\xi}\right\|^{2}\left(1+D_{\delta}\right)+\left\|\dot{e}_{\xi}\right\|^{2}+\dot{\gamma}_{i}\left(\gamma_{i}-\gamma_{i}^{*}\right) .
$$

Substituting (17) in (23) results in:

$$
\begin{array}{r}
\dot{V}_{i}<\bar{\Xi}\left\|e_{\xi}\right\|+\left\|e_{\xi}\right\|^{2}\left(1+D_{\delta}\right)+\left\|\dot{e}_{\xi}\right\|^{2}+\dot{\gamma}_{i}\left(\gamma_{i}-\gamma_{i}^{*}\right) \\
-e_{\xi}^{T}\left(\frac{\partial \hat{\xi}}{\partial c_{\xi}^{r}} \dot{c}_{\xi}^{r}+\frac{\partial \hat{\xi}}{\partial \omega_{1 \xi}^{r}} \dot{\omega}_{1 \xi}^{r}+\frac{\partial \hat{\xi}}{\partial \omega_{2 \xi}^{r}} \dot{\omega}_{2 \xi}^{r}+\frac{\partial \hat{\xi}}{\partial \xi^{*}} \dot{\xi}^{*}\right)
\end{array}
$$

Now comparing (16) and (24) gives:

$$
\begin{gathered}
\dot{V}_{i}<\bar{\Xi}\left\|e_{\xi}\right\|+\left\|e_{\xi}\right\|^{2}\left(1+D_{\delta}\right)+\left\|\dot{e}_{\xi}\right\|^{2}+\dot{\gamma}_{i}\left(\gamma_{i}-\gamma_{i}^{*}\right) \\
+\frac{\partial E_{i}}{\partial c_{\xi}^{r}} \dot{c}_{\xi}^{r}+\frac{\partial E_{i}}{\partial \omega_{1 \xi}^{r}} \dot{\omega}_{1 \xi}^{r}+\frac{\partial E_{i}}{\partial \omega_{2 \xi}^{r}} \dot{\omega}_{2 \xi}^{r}-e_{\xi}^{T} \frac{\partial \hat{\xi}}{\partial \xi^{*}} \dot{\xi}^{*}
\end{gathered}
$$

Substituting the proposed adaptive laws (14) and (15) in (25) and setting $\gamma_{i}^{*}>\max \left\{\bar{\Xi},\left(1+D_{\delta}\right)\right\}$

$$
\begin{aligned}
\dot{V}_{i}< & -(\underbrace{-\gamma_{i}}_{a_{c}}\left(\frac{\partial E_{i}}{\partial c_{\xi}^{r}}\right)^{2}+\underbrace{\left(1-\alpha_{i}\right) \partial c_{\xi}^{r}}_{b_{c}} \frac{\partial E_{i}}{\partial c_{\xi}^{r}} \underbrace{\left\|\dot{e}_{\xi}\right\|^{2}+e_{\xi}^{T} \frac{\partial \hat{\xi}}{\partial \xi^{*}} \dot{\xi}^{*}}_{c_{c}}) \\
& -(\underbrace{-\gamma_{i}}_{a_{\omega 1}}\left(\frac{\partial E_{i}}{\partial \omega_{1 \xi}^{r}}\right)^{2}+\underbrace{\left(1-\alpha_{i}\right) \omega_{1 \xi}^{r}}_{b_{\omega 1}} \frac{\partial E_{i}}{\partial \omega_{1 \xi}^{r}} \underbrace{-\gamma_{i}\left\|e_{\xi}\right\|^{2}}_{c_{\omega 1}}) \\
& -(\underbrace{-\gamma_{i}}_{a_{\omega 2}}\left(\frac{\partial E_{i}}{\partial \omega_{2 \xi}^{r}}\right)^{2}+\underbrace{\left(1-\alpha_{i}\right) \omega_{2 \xi}^{r}}_{b_{\omega 2}} \frac{\partial E_{i}}{\partial \omega_{2 \xi}^{r}} \underbrace{-\gamma_{i}\left\|e_{\xi}\right\|}_{c_{\omega 2}})
\end{aligned}
$$

which includes three quadratic equations:

$$
\dot{V}_{i}<-\left(\frac{\partial E_{i}}{\partial c_{\xi}^{r}}+P_{i}\right)^{2}-\left(\frac{\partial E_{i}}{\partial \omega_{1 \xi}^{r}}+N_{i}\right)^{2}-\left(\frac{\partial E_{i}}{\partial \omega_{2 \xi}^{r}}+Q_{i}\right)^{2}
$$

and makes the time-derivative of the Lyapunov-Krasovskii functional negative $\left(\dot{V}_{i}<0\right) . P_{i}, N_{i}$ and $Q_{i}$ are solutions of the quadratic equations that requires

$$
\begin{aligned}
& b_{c}^{2}-4 a_{c} c_{c}=0 \\
& b_{\omega 1}^{2}-4 a_{\omega 1} c_{\omega 1}=0 \\
& b_{\omega 2}^{2}-4 a_{\omega 2} c_{\omega 2}=0
\end{aligned}
$$

Solving (27) obtains two solutions for each parameter

$$
\begin{gathered}
\left(c_{\xi}^{r}\right)_{1,2}= \pm \sqrt{\frac{4 \gamma_{i}\left(e_{\xi}^{T} \frac{\partial \hat{\xi}}{\partial \xi^{*}} \dot{\xi}^{*}-\left\|\dot{e}_{\xi}\right\|^{2}\right)}{\left(1-\alpha_{i}\right)^{2}}} \\
\left(\omega_{1 \xi}^{r}\right)_{1,2}= \pm \sqrt{\frac{4 \gamma_{i}^{2}\left\|e_{\xi}\right\|^{2}}{\left(1-\alpha_{i}\right)^{2}}},\left(\omega_{2 \xi}^{r}\right)_{1,2}= \pm \sqrt{\frac{4 \gamma_{i}^{2}\left\|e_{\xi}\right\|}{\left(1-\alpha_{i}\right)^{2}}}
\end{gathered}
$$

and therefore, calculating $\left(-b \pm \sqrt{b^{2}-4 a c}\right) / 2 a$ for each $P_{i}, N_{i}$ and $Q_{i}$ results in

$$
\begin{gathered}
P_{i 1}=\frac{\left(1-\alpha_{i}\right)\left(c_{\xi}^{r}\right)_{1}}{\gamma_{i}}, P_{i 2}=\frac{\left(1-\alpha_{i}\right)\left(c_{\xi}^{r}\right)_{2}}{\gamma_{i}} \\
N_{i 1}=\frac{\left(1-\alpha_{i}\right)\left(\omega_{1 \xi}^{r}\right)_{1}}{\gamma_{i}}, N_{i 2}=\frac{\left(1-\alpha_{i}\right)\left(\omega_{1 \xi}^{r}\right)_{2}}{\gamma_{i}} \\
Q_{i 1}=\frac{\left(1-\alpha_{i}\right)\left(\omega_{2 \xi}^{r}\right)_{1}}{\gamma_{i}}, Q_{i 2}=\frac{\left(1-\alpha_{i}\right)\left(\omega_{2 \xi}^{r}\right)_{2}}{\gamma_{i}}
\end{gathered}
$$

and finally, (19) makes $\dot{V}_{i}$ much negative that guarantees the asymptotic convergence of the learning algorithms, and subsequently, stability of the proposed T2FNN controller for the both master and slave systems.

Remark1. It should be noted that the considered control signals are the desired position and velocity $\chi_{s}$ for the slave robot, and the desired haptic signal $\mathbf{f}_{h}$ for the master system, respectively. This is because the considered haptic device is capable to expose any demanded force to the human operator. On the other side, the slave robot is able to reach any desired, non-singular, configuration. Therefore, the teleoperation problem is providing the either systems with their corresponding desired references. 


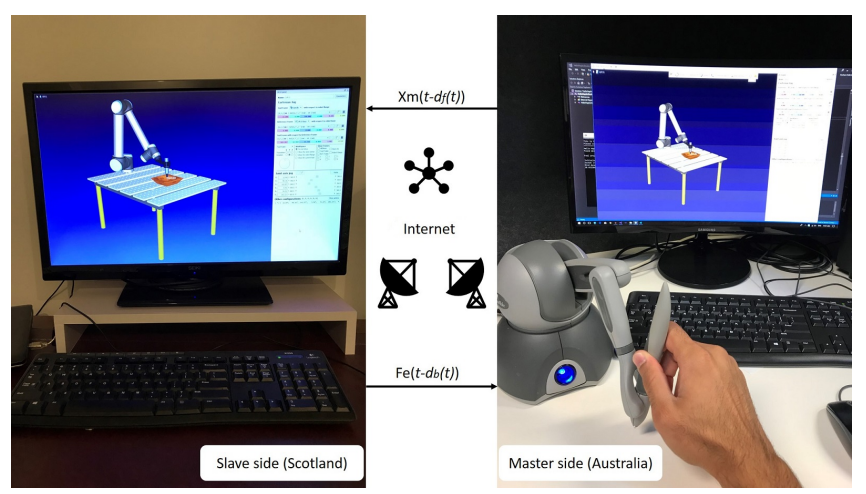

Fig. 7. The target teleoperation consisting of a virtual dynamic model of UR5 [73] as its slave teleoperator. Notably, the sampling rate of the simulation and control implementation is $1 \mathrm{kHz}$. This setup is used for evaluating the proposed adaptive T2FNN controller between Australia and Scotland [56].

\section{EXPERIMENTAL COMPARISON STUDY}

This section evaluates the performance of the proposed adaptive T2FNN control strategy on the intercontinental teleoperation setup, Figure 7. The main focus of this study is to experimentally assess and compare the performance of the proposed control method in this paper and those of [53] and [54] under a realistic teleoperation configuration. To this end, the master device is located in Australia while the slave robot is a full-scale SimMechanics model of the UR5 manipulator in Scotland. This model is developed based on the dynamical parameters and features reported by the manufacturer and previously developed in [73]. Moreover, the learning parameters $\alpha_{i}$ are identically considered as $10^{-4}$ for both the master and slave controllers. However, the adaptive learning rates $\gamma_{i}$ are shown in Figure 14. The communication between the master and slave sites are established through the Internet. Delays between the two sites of the teleoperation has been experimentally measured and the average experiences are depicted in Figure 8. Figure 8a illustrates the average of experienced delay through the forward channel and mean and standard deviation values of its distribution is also shown. Figure $8 \mathrm{~b}$ presents the same information for the backward channel.

The teleoperation task is considered in two phases including free motion and contact modes indicated in force reflection of the controllers (Figures 13a-13c). In the free motion genre, the human operator freely manoeuvres the master device in its workspace. Whilst, in the second phase, the slave robot is desired to vertically interact with an object, that introduces contact forces to the robot end-effector. As can be observed in those figures, the haptic signal provided by the adaptive T2FNN technique is much accurate than the other controllers. Comparing the tracking performance of the controllers in Figures $9 \mathrm{a}-9 \mathrm{c}$ for position, 10a-10c for orientation, and $11 \mathrm{a}-11 \mathrm{c}$ for velocity, the proposed adaptive T2FNN control methodology outperforms the other T2F based controllers. Torque signals generated by the slave robot under the three controllers are shown in Figures 12a-12c. Reported in Table I, values of root mean square error (RMSE), mean absolute error (MAE), and normalised RMSE (NRMSE) of the tracking errors of the
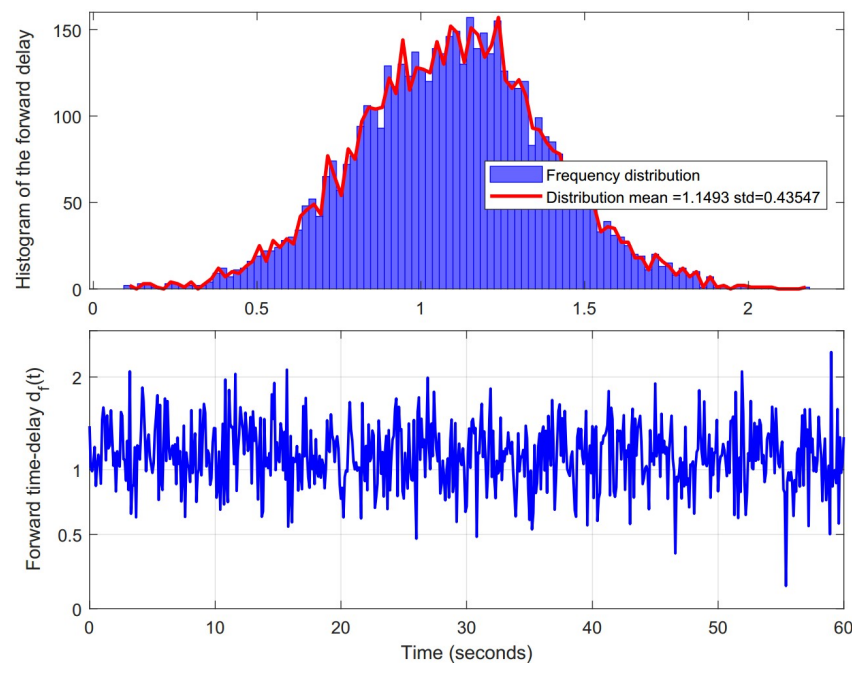

(a) Forward time-delay $d_{f}(t)$.
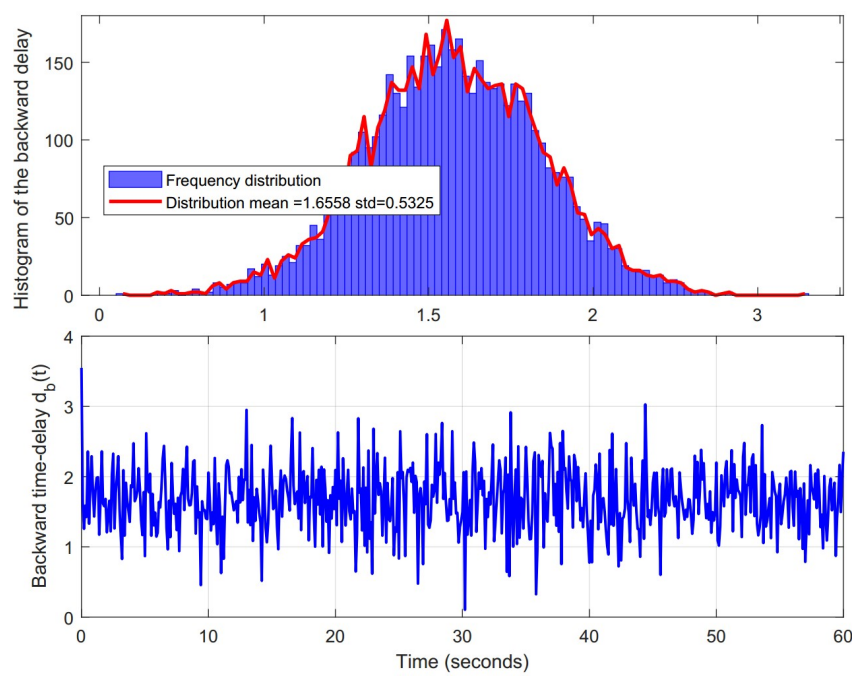

(b) Backward time-delay $d_{b}(t)$.

Fig. 8. Average forward and backward time-delays experimentally measured between Australia and Scotland.

proposed adaptive T2FNN controller are significantly smaller than those of the other T2F control algorithms. Considering NRMSE values, the proposed adaptive T2FNN controller has chiefly resulted in less numbers except in few cases, where the difference is ignorable. Additionally, to the best knowledge of the authors based on the literature, precise force reflection and practical haptic is one of the most critical challenges and objectives in teleoperation applications. On the other hand, it should be noted that the physical interaction between the slave robot and not-fully-known objects in the remote workspace is one of the major sources of uncertainties in teleoperation processes. Therefore, the superiority of the proposed approach in accurate force tracking is critically advantageous that is not achieved by many other solutions. Finally, Figure 14 illustrates the learning trend of the T2F parameters of both the master and slave controllers over 100 epochs considered for the training process. It should be noted that the training time is not reported since all the three methods took almost the same time. 


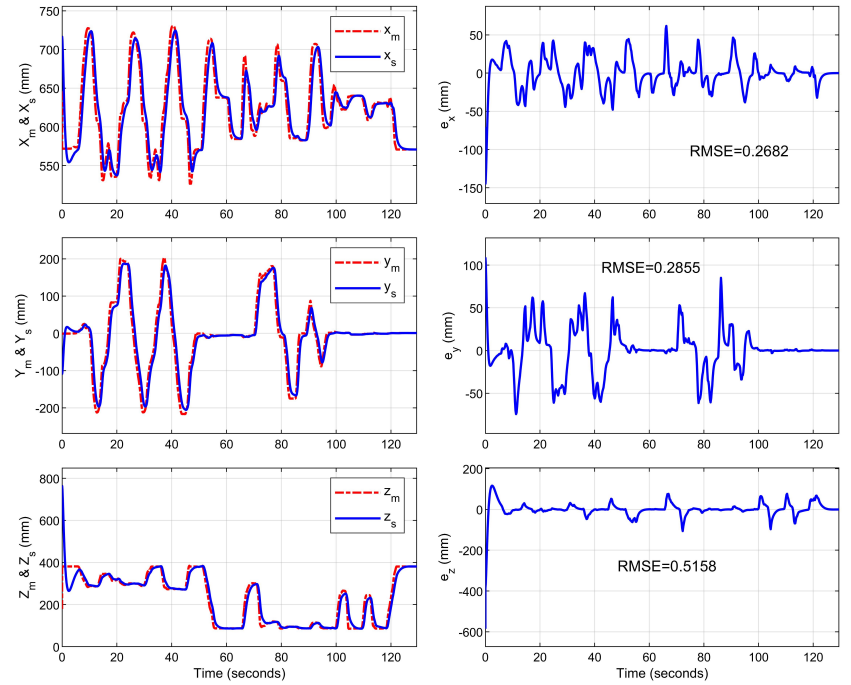

(a) Our proposed adaptive T2FNN controller.

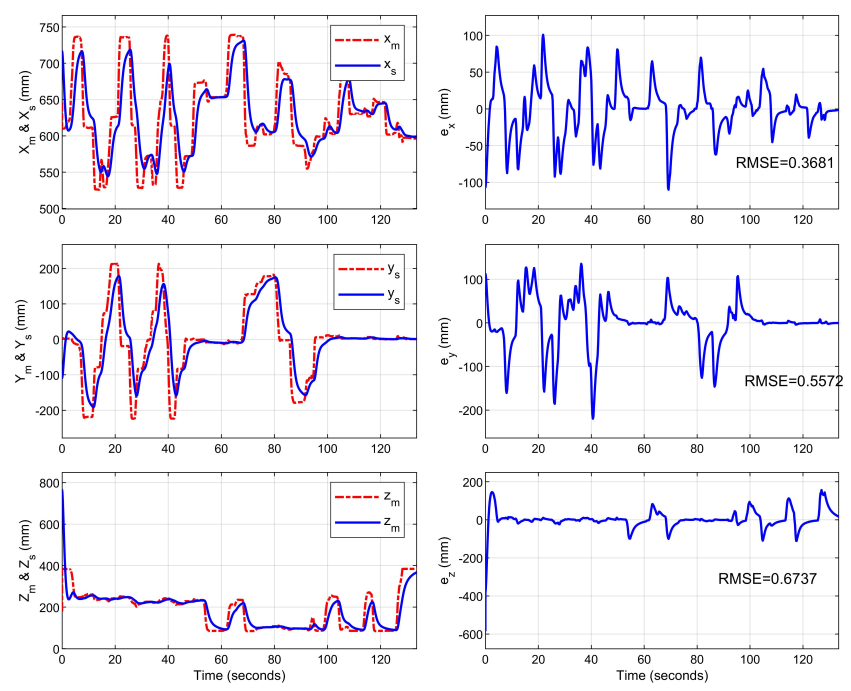

(b) The proposed controller in [53].
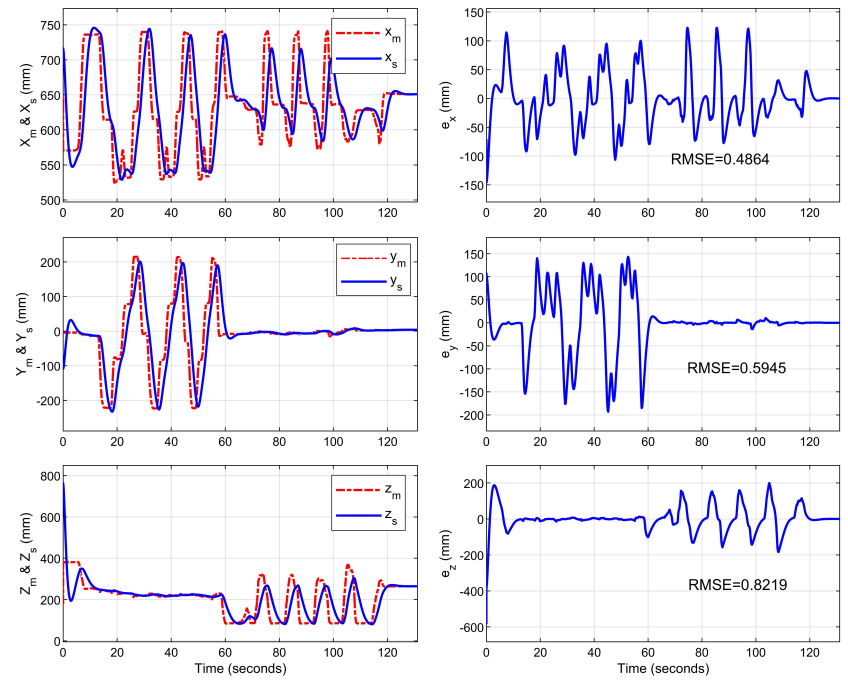

(c) The proposed controller in [54].

Fig. 9. Position tracking of the T2F based controllers.
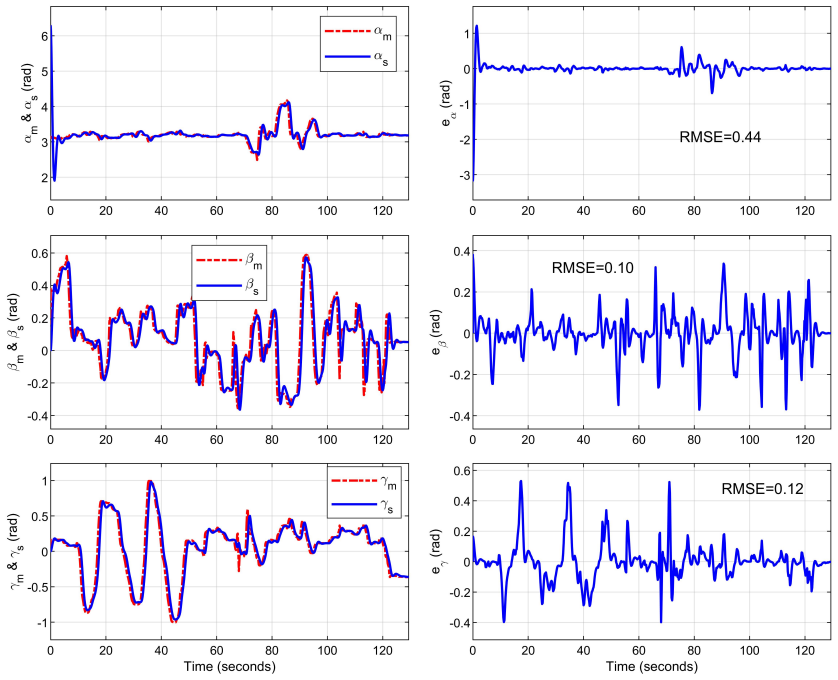

(a) Our proposed adaptive T2FNN controller.
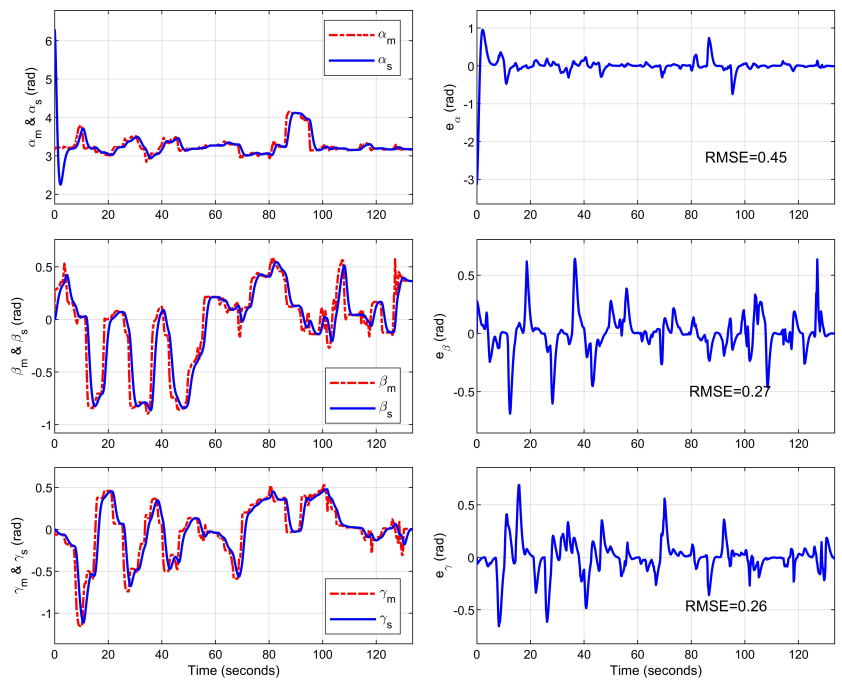

(b) The proposed controller in [53].
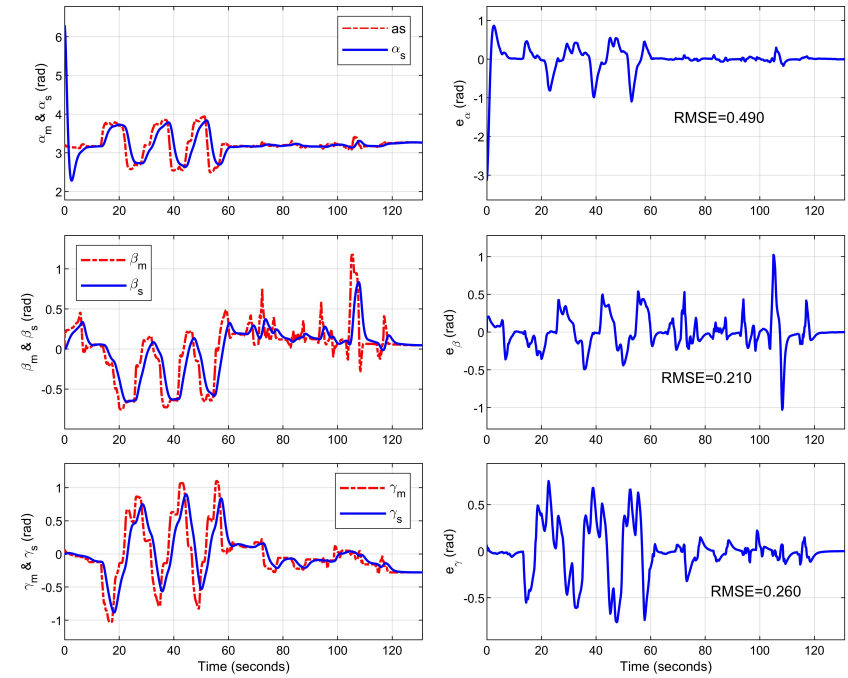

(c) The proposed controller in [54].

Fig. 10. Orientation tracking of the T2F based controllers. 


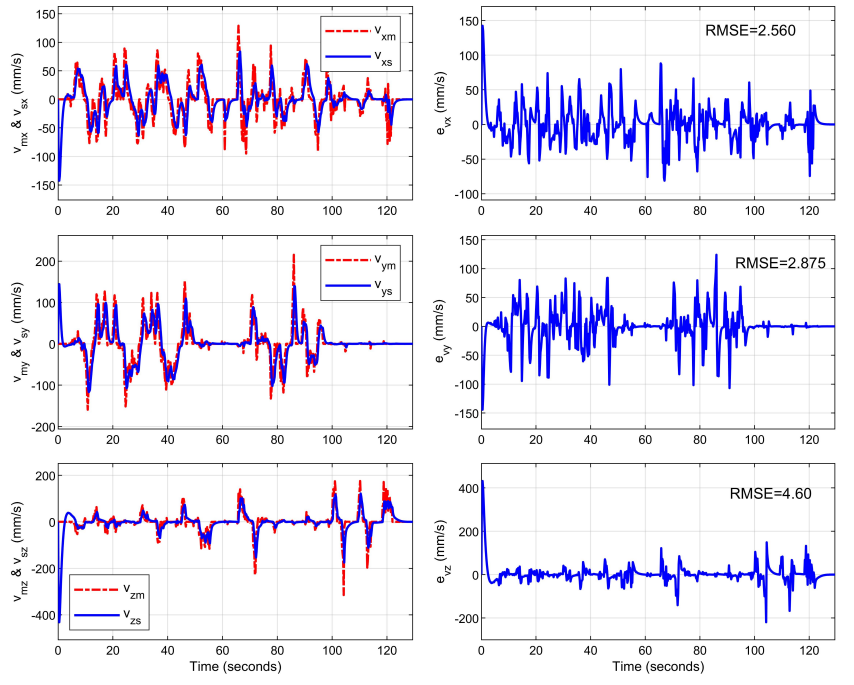

(a) Our proposed adaptive T2FNN controller.
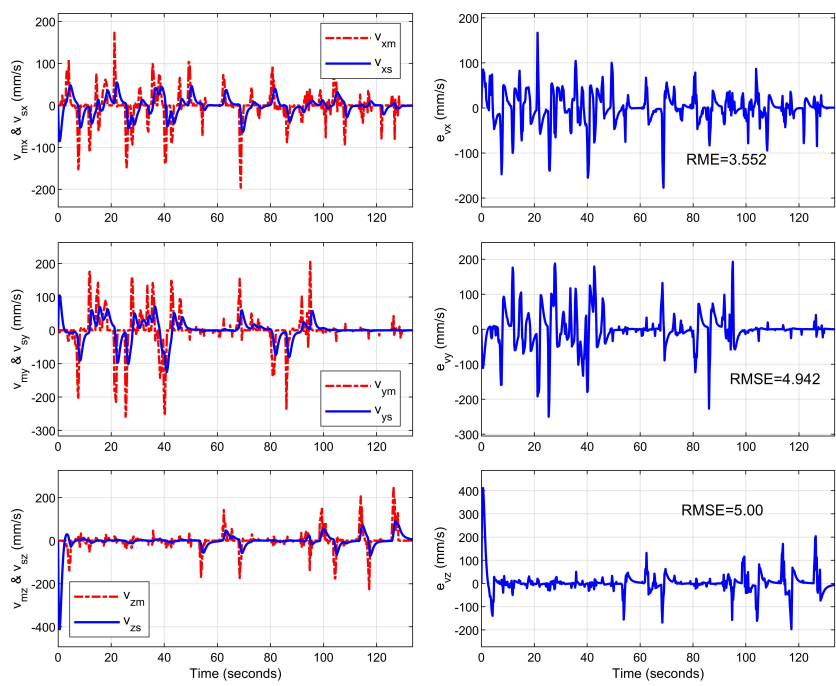

(b) The proposed controller in [53].
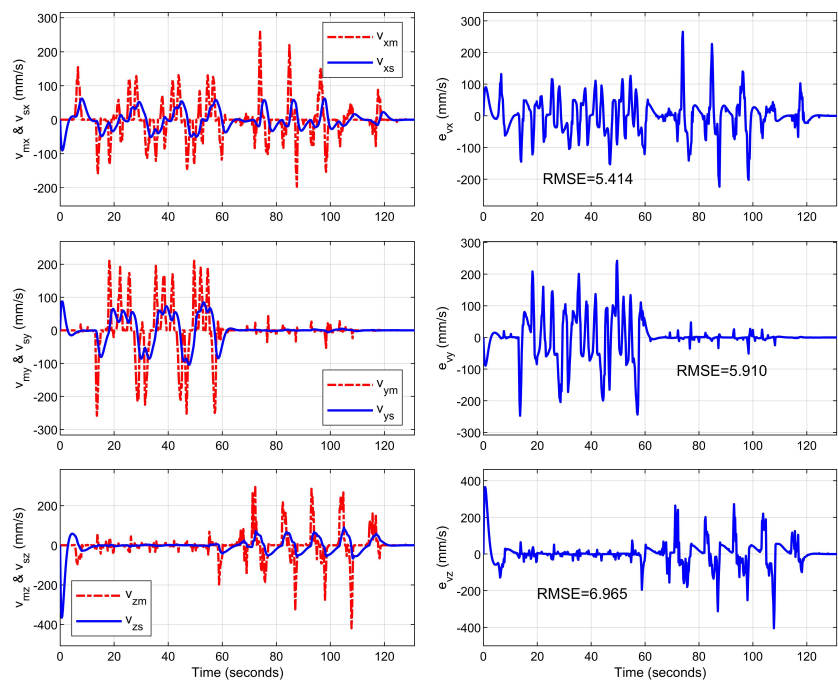

(c) The proposed controller in [54].

Fig. 11. Velocity tracking of the T2F based controllers.

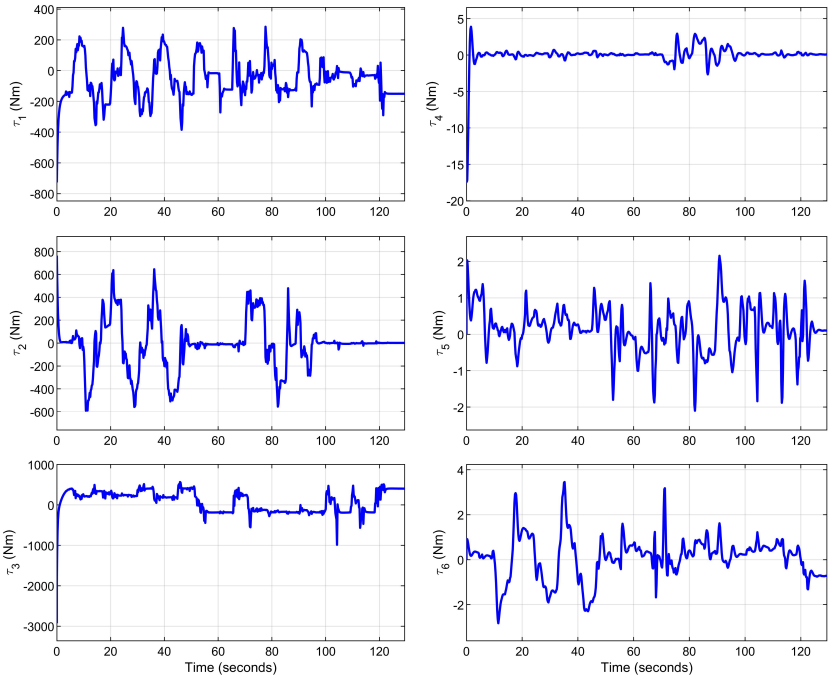

(a) Our proposed adaptive T2FNN controller.

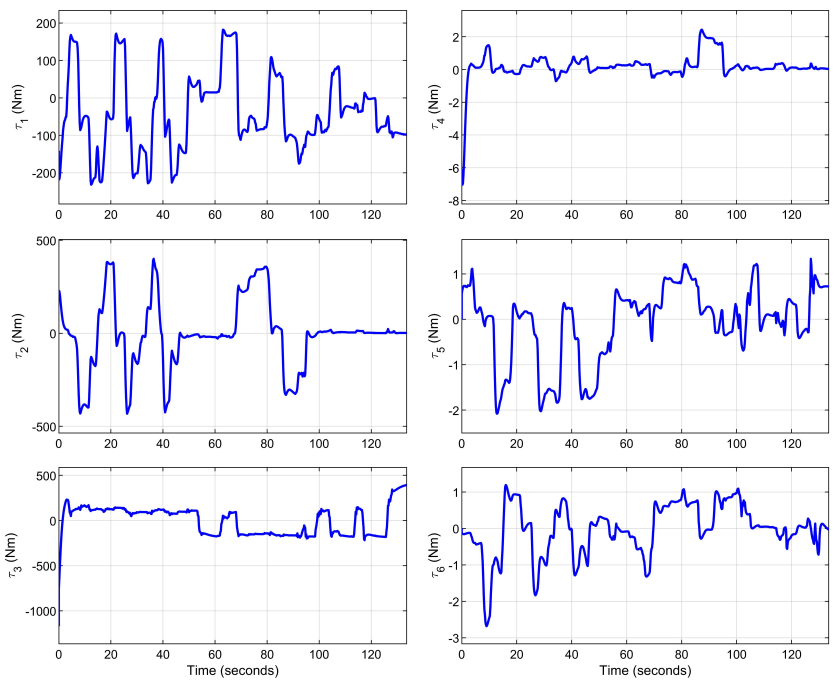

(b) The proposed controller in [53].
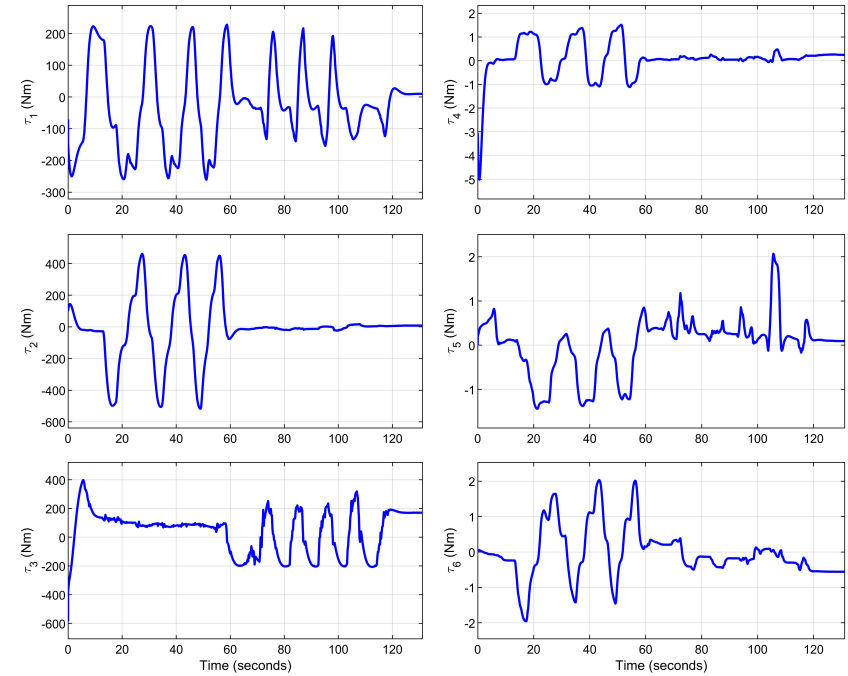

(c) The proposed controller in [54].

Fig. 12. Slave's torques of the T2F based controllers. 
TABLE I

RMSE, MAE AND NRMSE VALUES OF THE THREE T2F-BASED CONTROLLERS. THE PROPOSED ADAPTIVE T2FNN CONTROL ALGORITHM HAS OUTPERFORMED THE OTHER TWO APPROACHES [53] AND [54].

\begin{tabular}{|c|c||c|c|c||c|c|c||c|c|c|}
\hline \multicolumn{2}{|c|}{ Metrics: } & \multicolumn{3}{|c||}{ RMSE } & \multicolumn{3}{c||}{ MAE } & \multicolumn{3}{c|}{ NRMSE } \\
\hline \multicolumn{2}{|c|}{ Methods: } & AT2FNN & {$[53]$} & {$[54]$} & AT2FNN & {$[53]$} & {$[54]$} & AT2FNN & {$[53]$} & {$[54]$} \\
\hline \multirow{3}{*}{ Position } & $x$ & $\mathbf{0 . 2 6 8 2}$ & 0.3681 & 0.4864 & $\mathbf{1 6 . 0 3 8 7}$ & 25.7914 & 34.6060 & $\mathbf{0 . 1 2 9 9}$ & 0.1746 & 0.1809 \\
& $y$ & $\mathbf{0 . 2 8 5 5}$ & 0.5572 & 0.5946 & $\mathbf{1 7 . 5 9 9 2}$ & 35.5523 & 33.9617 & $\mathbf{0 . 1 5 5 7}$ & 0.1569 & 0.1767 \\
& $z$ & $\mathbf{0 . 5 1 5 8}$ & 0.6737 & 0.8219 & $\mathbf{2 3 . 7 6 4 1}$ & 30.8428 & 44.6107 & $\mathbf{0 . 0 8 7 8}$ & 0.0908 & 0.1047 \\
\hline \multirow{3}{*}{ Orientation } & $\alpha$ & $\mathbf{0 . 4 4}$ & 0.45 & 0.490 & $\mathbf{0 . 1 1 6 0}$ & 0.1410 & 0.1963 & $\mathbf{0 . 1 0 1 5}$ & 0.1100 & 0.1216 \\
& $\beta$ & $\mathbf{0 . 1 0}$ & 0.27 & 0.210 & $\mathbf{0 . 0 6 4 9}$ & 0.1064 & 0.1413 & $\mathbf{0 . 1 0 3 7}$ & 0.1242 & 0.1387 \\
& $\gamma$ & $\mathbf{0 . 1 2}$ & 0.26 & 0.260 & $\mathbf{0 . 0 7 5 8}$ & 0.1014 & 0.1632 & 0.1328 & $\mathbf{0 . 1 1 9 7}$ & 0.1685 \\
\hline \multirow{3}{*}{ Velocity } & $v_{x}$ & $\mathbf{2 . 5 6 0}$ & 3.552 & 5.414 & $\mathbf{1 6 . 9 8 8 4}$ & 23.0686 & 36.6404 & 0.1114 & $\mathbf{0 . 1 0 3 7}$ & 0.1107 \\
& $v_{y}$ & $\mathbf{2 . 8 7 5}$ & 4.942 & 5.910 & $\mathbf{1 6 . 3 6 3 4}$ & 28.4500 & 32.2861 & $\mathbf{0 . 1 0 7 1}$ & 0.1116 & 0.1208 \\
& $v_{z}$ & $\mathbf{4 . 6 0}$ & 5.00 & 6.965 & $\mathbf{2 0 . 1 0 0 7}$ & 22.4033 & 37.5394 & $\mathbf{0 . 0 7 0 6}$ & 0.0821 & 0.0904 \\
\hline Force & $f_{z}$ & $\mathbf{0 . 2 6 2 4}$ & 0.563 & 0.5029 & $\mathbf{0 . 0 9 0 3}$ & 0.2767 & 0.2445 & $\mathbf{0 . 0 9 9 2}$ & 0.1215 & 0.1349 \\
\hline
\end{tabular}

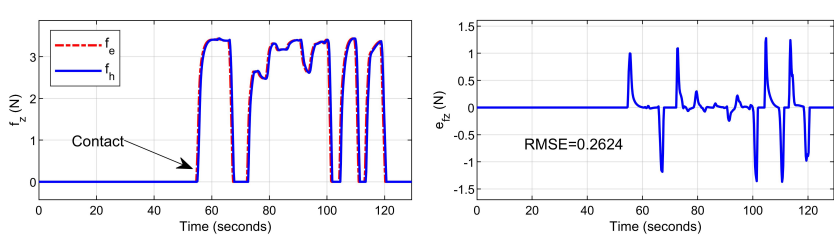

(a) Our proposed adaptive T2FNN controller.
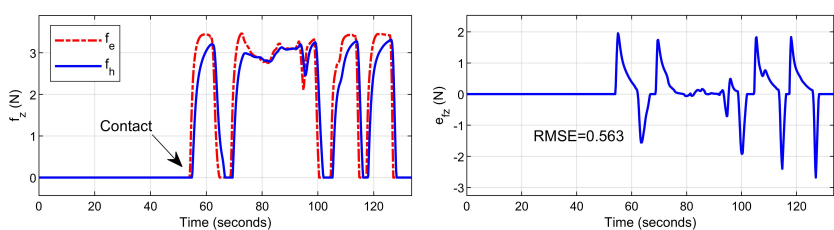

(b) The proposed controller in [53].
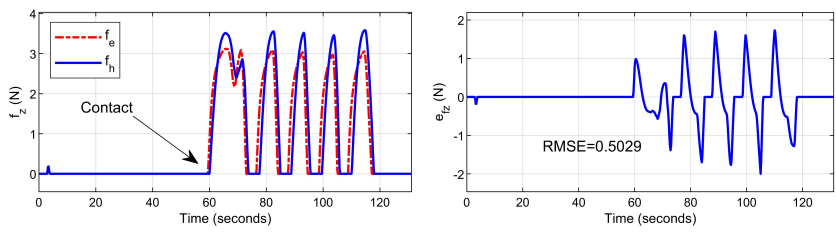

(c) The proposed controller in [54].

Fig. 13. Force reflection of the T2F based controllers.

\section{Conclusions}

This study addressed the problem of teleoperation under time-varying delays and uncertainties. Known as one of the strongest tools in dealing with uncertainties, Type2 fuzzy approach is utilised to handle this issue in teleoperation applications. Moreover, artificial neural networks are employed to further strengthen the nonlinear modelling capabilities of the proposed algorithm. Additionally, errorbased adaptive laws are developed for tuning parameters of the T2FNN controller to improve the overall performance of the teleoperation system. Proven by Lyapunov-Krasovskii methodology, the proposed online updating algorithm does not get trapped in local minima. Notably, the T2FNN has learned on the training data collected by a simple teleoperation setup shown in Figure 1. However, the resulted control technique is then applied and verified on a completely different and more

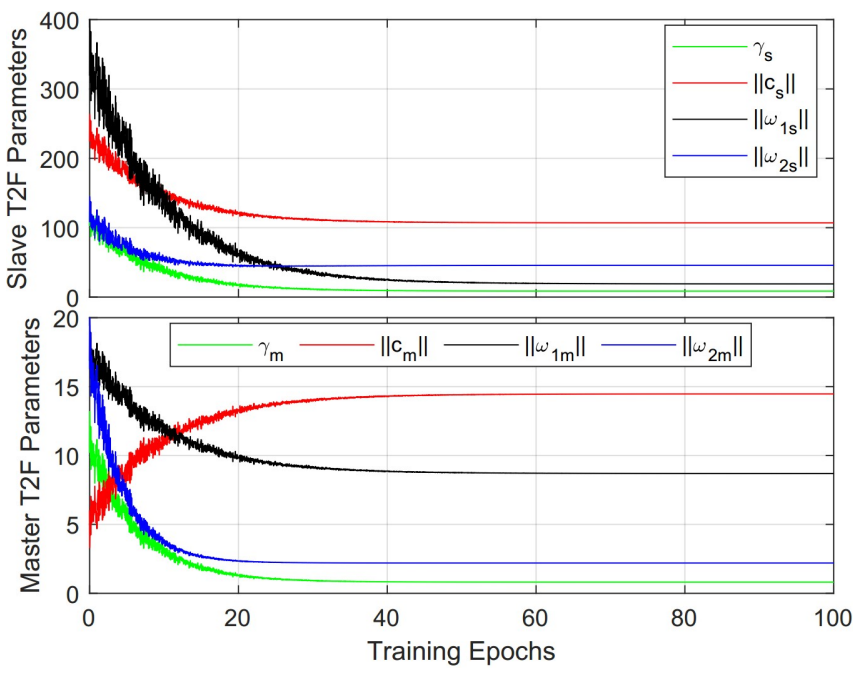

Fig. 14. Norm value of T2F parameters of the master and slave controllers.

complicated teleoperation configuration. Demonstrated in the comparative experimental simulations, the proposed adaptive T2FNN control strategy outperformed the most recent T2F based controllers in the literature. The RMSE, MAE, and NRMSE values of the three T2F-based controllers have been also reported to better compare the performance the three controllers.

In the future, further investigations on the T2FNN structures are being considered for semi/autonomous teleoperative assistance to the human operator. Moreover, considering adjustable parameters in fuzzy rules, including $a_{i n}^{r}, i \in\{m, s\}$ is a future direction to further improve the performance of the system. Another strategy might be considered as development of nonlinear functions for the consequent parts of type-2 fuzzy rules. Chiefly, intelligent predictive models of the teleoperation system will be developed that autonomously executes secondary tasks alongside the main teleoperation process.

\section{REFERENCES}

[1] Y. Gao and S. Chien, "Review on space robotics: Toward top-level science through space exploration," Science Robotics, vol. 2, no. 7, pp. 1-11, 2017. 
[2] B. T. Phillips, K. P. Becker, S. Kurumaya, K. C. Galloway, G. Whit tredge, D. M. Vogt, C. B. Teeple, M. H. Rosen, V. A. Pieribone, D. F. Gruber et al., "A dexterous, glove-based teleoperable low-power soft robotic arm for delicate deep-sea biological exploration," Scientific reports, vol. 8, no. 14779, pp. 1-11, 2018.

[3] A. Shademan, R. S. Decker, J. D. Opfermann, S. Leonard, A. Krieger, and P. C. Kim, "Supervised autonomous robotic soft tissue surgery," Science translational medicine, vol. 8, no. 337, p. 337ra64, 2016.

[4] H.-H. Liu, L.-J. Li, B. Shi, C.-W. Xu, and E. Luo, "Robotic surgical systems in maxillofacial surgery: a review," International journal of oral science, vol. 9, no. 2, pp. 63-73, 2017.

[5] M. M. Dalvand, S. Nahavandi, M. Fielding, J. Mullins, Z. Najdovski, and R. D. Howe, "Modular instrument for a haptically-enabled robotic surgical system (herosurg)," IEEE Access, vol. 6, pp. 31974-31982, 2018.

[6] P. F. Hokayem and M. W. Spong, "Bilateral teleoperation: An historical survey," Automatica, vol. 42, no. 12, pp. 2035-2057, 2006.

[7] S. Hirche and M. Buss, "Human-oriented control for haptic teleoperation," Proceedings of the IEEE, vol. 100, no. 3, pp. 623-647, March 2012.

[8] P. M. Kebria, H. Abdi, M. M. Dalvand, A. Khosravi, and S. Nahavandi, "Control methods for internet-based teleoperation systems: A review," IEEE Transactions on Human-Machine Systems, vol. 49, no. 1, pp. 32 46, Feb 2019.

[9] L. A. Zadeh, "Fuzzy sets," Information and Control, no. 8, pp. 338-353, 1965.

[10] - , "A fuzzy-algorithmic approach to the definition of complex or imprecise concepts," in Systems Theory in the Social Sciences. Springer, 1976, pp. 202-282.

[11] — "Fuzzy logic," Computer, no. 21, pp. 83-93, 1988.

[12] - "Fuzzy logic= computing with words," IEEE Transactions on Fuzzy Systems, vol. 4, no. 2, pp. 103-111, 1996.

[13] - "Outline of a new approach to the analysis of complex systems and decision processes," IEEE Transactions on systems, Man, and Cybernetics, no. 1, pp. 28-44, 1973.

[14] M. Sugeno, "An introductory survey of fuzzy control," Information sciences, vol. 36, no. 1-2, pp. 59-83, 1985.

[15] L.-X. Wang and J. M. Mendel, "Fuzzy basis functions, universal approximation, and orthogonal least-squares learning," IEEE transactions on Neural Networks, vol. 3, no. 5, pp. 807-814, 1992.

[16] P. Melin, E. Ontiveros-Robles, C. I. Gonzalez, J. R. Castro, and O. Castillo, "An approach for parameterized shadowed type-2 fuzzy membership functions applied in control applications," Soft Computing, vol. 23 , no. 11 , pp. 3887-3901, 2019.

[17] L. Cervantes and O. Castillo, "Type-2 fuzzy logic aggregation of multiple fuzzy controllers for airplane flight control," Information Sciences vol. 324, pp. 247-256, 2015.

[18] M. A. Sanchez, O. Castillo, and J. R. Castro, "Generalized type-2 fuzzy systems for controlling a mobile robot and a performance comparison with interval type-2 and type-1 fuzzy systems," Expert Systems with Applications, vol. 42, no. 14, pp. 5904-5914, 2015.

[19] R. H. Abiyev and O. Kaynak, "Type 2 fuzzy neural structure for identification and control of time-varying plants," IEEE Transaction on Industrial Electronics, vol. 57, no. 12, pp. 4147-4159, 2010.

[20] W. Shi, "Adaptive fuzzy control for mimo nonlinear systems with nonsymmetric control gain matrix and unknown control direction," IEEE Transactions on Fuzzy Systems, vol. 22, no. 5, pp. 1288-1300, 2013

[21] N. A. Sofianos and Y. S. Boutalis, "Stable indirect adaptive switching control for fuzzy dynamical systems based on t-s multiple models," International Journal of Systems Science, vol. 44, no. 8, pp. 1546-1565, 2013.

[22] $ـ$, "Robust adaptive multiple models based fuzzy control of nonlinear systems," Neurocomputing, vol. 173, pp. 1733-1742, 2016.

[23] A. Khosravi, S. Nahavandi, and D. Creighton, "Short term load forecasting using interval type-2 fuzzy logic systems," in Fuzzy Systems (FUZZ), 2011 IEEE International Conference on. IEEE, 2011, pp. 502-508.

[24] A. Khosravi, S. Nahavandi, D. Creighton, and R. Naghavizadeh, "Prediction interval construction using interval type-2 fuzzy logic systems," in Fuzzy Systems (FUZZ-IEEE), 2012 IEEE International Conference on. IEEE, 2012, pp. 1-7.

[25] A. Khosravi, S. Nahavandi, D. Creighton, and D. Srinivasan, "Interval type-2 fuzzy logic systems for load forecasting: A comparative study," IEEE Transactions on Power Systems, vol. 27, no. 3, pp. 1274-1282, 2012.

[26] A. Khosravi and S. Nahavandi, "Load forecasting using interval type2 fuzzy logic systems: Optimal type reduction," IEEE Transactions on Industrial Informatics, vol. 10, no. 2, pp. 1055-1063, 2014
[27] T. Takagi and M. Sugeno, "Fuzzy identification of systems and its applications to modeling and control," IEEE transactions on systems, man, and cybernetics, no. 1, pp. 116-132, 1985.

[28] K. Zeng, N.-Y. Zhang, and W.-L. Xu, "A comparative study on sufficient conditions for takagi-sugeno fuzzy systems as universal approximators," IEEE Transactions on fuzzy systems, vol. 8, no. 6, pp. 773-780, 2000.

[29] G. Feng, "A survey on analysis and design of model-based fuzzy control systems," IEEE Transactions on Fuzzy systems, vol. 14, no. 5, pp. 676697, 2006.

[30] E. Ontiveros-Robles, P. Melin, and O. Castillo, "Comparative analysis of noise robustness of type 2 fuzzy logic controllers," Kybernetika, vol. 54, no. 1 , pp. 175-201, 2018.

[31] E. H. Mamdani, "Application of fuzzy algorithms for control of simple dynamic plant," in Proceedings of the institution of electrical engineers, vol. 121, no. 12. IET, 1974, pp. 1585-1588.

[32] C.-C. Lee, "Fuzzy logic in control systems: fuzzy logic controller. i," IEEE Transactions on systems, man, and cybernetics, vol. 20, no. 2, pp. 404-418, 1990.

[33] H. A. Hagras, "A hierarchical type-2 fuzzy logic control architecture for autonomous mobile robots," IEEE Transactions on Fuzzy systems, vol. 12 , no. 4 , pp. 524-539, 2004

[34] N. N. Karnik and J. M. Mendel, "Introduction to type-2 fuzzy logic systems," in Fuzzy Systems Proceedings, 1998. IEEE World Congress on Computational Intelligence., The 1998 IEEE International Conference on, vol. 2. IEEE, 1998, pp. 915-920.

[35] N. N. Karnik, J. M. Mendel, and Q. Liang, "Type-2 fuzzy logic systems," IEEE transactions on Fuzzy Systems, vol. 7, no. 6, pp. 643-658, 1999.

[36] Q. Liang and J. M. Mendel, "Interval type-2 fuzzy logic systems: theory and design," IEEE Transactions on Fuzzy systems, vol. 8, no. 5, pp. 535$550,2000$.

[37] $\mathrm{H}$. Wu and J. M. Mendel, "Uncertainty bounds and their use in the design of interval type-2 fuzzy logic systems," IEEE Transactions on fuzzy systems, vol. 10, no. 5, pp. 622-639, 2002.

[38] J. M. Mendel and R. I. B. John, "Type-2 fuzzy sets made simple," IEEE Transactions on Fuzzy Systems, vol. 10, no. 2, pp. 117-127, April 2002.

[39] J. M. Mendel, R. I. John, and F. Liu, "Interval type-2 fuzzy logic systems made simple," IEEE transactions on fuzzy systems, vol. 14 , no. 6 , pp $808-821,2006$

[40] J. M. Mendel, "Type-2 fuzzy sets and systems: An overview [corrected reprint]," IEEE computational intelligence magazine, vol. 2, no. 2, pp. 20-29, 2007.

[41] - "Advances in type-2 fuzzy sets and systems," Information sciences, vol. 177, no. 1, pp. 84-110, 2007.

[42] O. Castillo, L. Amador-Angulo, J. R. Castro, and M. Garcia-Valdez, "A comparative study of type-1 fuzzy logic systems, interval type-2 fuzzy logic systems and generalized type-2 fuzzy logic systems in control problems," Information Sciences, vol. 354, pp. 257-274, 2016.

[43] K.-B. Sim, K.-S. Byun, and F. Harashima, "Internet-based teleoperation of an intelligent robot with optimal two-layer fuzzy controller," IEEE Transactions on Industrial Electronics, vol. 53, no. 4, pp. 1362-1372, 2006.

[44] F.-L. Chung and J.-C. Duan, "On multistage fuzzy neural network modeling," IEEE Transactions on fuzzy systems, vol. 8, no. 2, pp. 125 $142,2000$.

[45] Z. Li, Y. Xia, and F. Sun, "Adaptive fuzzy control for multilateral cooperative teleoperation of multiple robotic manipulators under random network-induced delays," IEEE Transactions on Fuzzy Systems, vol. 22, no. 2, pp. 437-450, 2014

[46] Y. Yang, C. Hua, and X. Guan, "Adaptive fuzzy finite-time coordination control for networked nonlinear bilateral teleoperation system," IEEE Transactions on Fuzzy Systems, vol. 22, no. 3, pp. 631-641, 2014.

[47] X. Yang, C.-C. Hua, J. Yan, and X.-P. Guan, "A new master-slave torque design for teleoperation system by ts fuzzy approach," IEEE Transactions on Control Systems Technology, vol. 23, no. 4, pp. 16111619, 2015.

[48] D.-H. Zhai and Y. Xia, "Adaptive fuzzy control of multilateral asymmetric teleoperation for coordinated multiple mobile manipulators," IEEE Transactions on Fuzzy Systems, vol. 24, no. 1, pp. 57-70, 2016.

[49] U. Farooq, J. Gu, M. El-Hawary, M. U. Asad, and G. Abbas, "Fuzzy model based bilateral control design of nonlinear tele-operation system using method of state convergence," IEEE Access, vol. 4, pp. 4119-4135, 2016.

[50] M. A. Khanesar, O. Kaynak, S. Yin, and H. Gao, "Adaptive indirect fuzzy sliding mode controller for networked control systems subject to time-varying network-induced time delay," IEEE Transactions on Fuzzy Systems, vol. 23, no. 1, pp. 205-214, 2015. 
[51] A. Nasirian and M. A. Khanesar, "Sliding mode fuzzy rule base bilateral teleoperation control of 2-dof scara system," in Automatic Control and Dynamic Optimization Techniques (ICACDOT), International Conference on. IEEE, 2016, pp. 7-12.

[52] Z. Lu, P. Huang, Z. Liu, and H. Chen, "Fuzzy observer-based hybrid force/position control design for a multiple-sampling-rate bimanual teleoperation system," IEEE Transactions on Fuzzy Systems, Early Access, pp. 1-15, 2018.

[53] S. Ganjefar and Y. Solgi, "A lyapunov stable type-2 fuzzy wavelet network controller design for a bilateral teleoperation system," Information Sciences, vol. 311, pp. 1-17, 2015.

[54] D. Sun, Q. Liao, and H. Ren, "Type-2 fuzzy modeling and control for bilateral teleoperation system with dynamic uncertainties and timevarying delays," IEEE Transactions on Industrial Electronics, vol. 65 no. 1, pp. 447-459, Jan 2018

[55] D. Sun, Q. Liao, X. Gu, C. Li, and H. Ren, "Multilateral teleoperation with new cooperative structure based on reconfigurable robots and type2 fuzzy logic," IEEE Transactions on Cybernetics, Early Access, no. 99 , pp. 1-15, 2018.

[56] P. M. Kebria, A. Khosravi, S. Nahavandi, P. Shi, and R. Alizadehsani, "Robust adaptive control scheme for teleoperation systems with delay and uncertainties," IEEE Transactions on Cybernetics, Early Access, pp. 1-11, 2019.

[57] P. M. Kebria, A. Khosravi, S. Nahavandi, D. Watters, G. Guest, and P. Shi, "Robust adaptive control of internet-based bilateral teleoperation systems with time-varying delay and model uncertainties," in 2019 IEEE International Conference on Industrial Technology (ICIT), Feb 2019, pp. 187-192.

[58] P. M. Kebria, A. Khosravi, S. Nahavandi, F. Bello, and S. Krishnan, "Robust adaptive synchronisation of a single-master multi-slave teleoperation system over delayed communication," in 2019 IEEE International Conference on Industrial Technology (ICIT), Feb 2019, pp. 193-198.

[59] C.-H. Wang, C.-S. Cheng, T.-T. Lee et al., "Dynamical optimal training for interval type-2 fuzzy neural network (t2fnn)," IEEE Transactions on Systems Man and Cybernetics Part B-Cybernetics, vol. 34, no. 3, pp. 1462-1477, 2004.

[60] C.-F. Juang and Y.-W. Tsao, "A self-evolving interval type-2 fuzzy neural network with online structure and parameter learning," IEEE Transactions on Fuzzy Systems, vol. 16, no. 6, pp. 1411-1424, 2008.

[61] J. R. Castro, O. Castillo, P. Melin, and A. Rodríguez-Díaz, "A hybrid learning algorithm for a class of interval type-2 fuzzy neural networks," Information Sciences, vol. 179, no. 13, pp. 2175-2193, 2009.

[62] F.-J. Lin, P.-H. Chou et al., "Adaptive control of two-axis motion control system using interval type-2 fuzzy neural network," IEEE Trans. Industrial Electronics, vol. 56, no. 1, pp. 178-193, 2009.

[63] O. Castillo and P. Melin, "A review on the design and optimization of interval type-2 fuzzy controllers," Applied Soft Computing, vol. 12, no. 4, pp. 1267-1278, 2012.

[64] Y.-Y. Lin, J.-Y. Chang, and C.-T. Lin, "A tsk-type-based self-evolving compensatory interval type-2 fuzzy neural network (tscit2fnn) and its applications," IEEE Transactions on Industrial Electronics, vol. 61, no. 1, pp. 447-459, 2014.

[65] Y.-Y. Lin, S.-H. Liao, J.-Y. Chang, C.-T. Lin et al., "Simplified interval type-2 fuzzy neural networks." IEEE Trans. Neural Netw. Learning Syst., vol. 25, no. 5, pp. 959-969, 2014.

[66] O. Castillo and P. Melin, "A review on interval type-2 fuzzy logic applications in intelligent control," Information Sciences, vol. 279, pp. 615-631, 2014.

[67] S. Hassan, M. A. Khanesar, E. Kayacan, J. Jaafar, and A. Khosravi, "Optimal design of adaptive type-2 neuro-fuzzy systems: A review," Applied Soft Computing, vol. 44, pp. 134-143, 2016.

[68] Z. Li and C.-Y. Su, "Neural-adaptive control of single-master-multipleslaves teleoperation for coordinated multiple mobile manipulators with time-varying communication delays and input uncertainties," IEEE transactions on neural networks and learning systems, vol. 24, no. 9 , pp. 1400-1413, 2013

[69] H. Wang, P. X. Liu, and S. Liu, "Adaptive neural synchronization control for bilateral teleoperation systems with time delay and backlash-like hysteresis," IEEE transactions on cybernetics, vol. 47, no. 10, pp. 3018 3026, 2017.

[70] D. Sun, F. Naghdy, and H. Du, "Neural network-based passivity control of teleoperation system under time-varying delays," IEEE Transactions on Cybernetics, vol. 47, no. 7, pp. 1666-1680, 2017.

[71] P. M. Kebria, A. Khosravi, S. Nahavandi, Z. Najdovski, and S. J. Hilton, "Neural network adaptive control of teleoperation systems with uncertainties and time-varying delay," in 2018 IEEE 14th International
Conference on Automation Science and Engineering (CASE), Aug 2018, pp. 252-257.

[72] P. M. Kebria, H. Abdi, and S. Nahavandi, "Development and evaluation of a symbolic modelling tool for serial manipulators with any number of degrees of freedom," in 2016 IEEE International Conference on Systems, Man, and Cybernetics (SMC), Oct 2016, pp. 4223-4228.

[73] P. M. Kebria, S. Al-wais, H. Abdi, and S. Nahavandi, "Kinematic and dynamic modelling of ur5 manipulator," in 2016 IEEE International Conference on Systems, Man, and Cybernetics (SMC), Oct 2016, pp. 4229-4234.

[74] P. M. Kebria, A. Khosravi, S. Nahavandi, A. Homaifar, and M. Saif "Experimental comparison study on joint and cartesian space control schemes for a teleoperation system under time-varying delay," in 2019 IEEE International Conference on Industrial Technology (ICIT), Feb 2019, pp. 108-113.

[75] M. Biglarbegian, W. W. Melek, and J. M. Mendel, "On the stability of interval type-2 tsk fuzzy logic control systems," IEEE Transactions on Systems, Man, and Cybernetics, Part B (Cybernetics), vol. 40, no. 3, pp. 798-818, 2009.

[76] L. Behera, S. Kumar, and A. Patnaik, "On adaptive learning rate that guarantees convergence in feedforward networks," IEEE transactions on neural networks, vol. 17, no. 5, pp. 1116-1125, 2006. 